\title{
On the Uniform Quantization of a Class of Sparse Sources
}

\author{
Aurélia Fraysse, Béatrice Pesquet-Popescu, Senior Member IEEE, and \\ Jean-Christophe Pesquet, Senior Member IEEE
}

\begin{abstract}
We consider the uniform scalar quantization of a class of mixed distributed memoryless sources, namely sources having a Bernoulli-Generalized Gaussian distribution. Both for low and high resolutions, asymptotic expressions of the distortion for a $p$-th order moment error measure, and close approximations of the entropy are provided for these sources. Operational rate-distortion functions at high bitrate and their slope factors at low bitrate are derived. The dependence of these results on $p$ and the distribution parameters as well as the relation to the Shannon optimal rate-distortion bound are then discussed.
\end{abstract}

\section{Index Terms}

Rate-distortion function, uniform quantization, asymptotic performance, generalized Gaussian, Bernoulli-Gaussian, mixed distribution, sparsity, transform coding.

\section{INTRODUCTION}

One commonly used probabilistic model is the generalized Gaussian (GG) model (sometimes called the exponential power model). In particular, it has been extensively employed [1]-[3] for modelling the distribution of sparse coefficients generated by the wavelet decomposition of regular signals. The corresponding probability density function is given by

$$
\forall \xi \in \mathbb{R}, \quad f(\xi)=\frac{\beta \omega^{1 / \beta}}{2 \Gamma(1 / \beta)} e^{-\omega|\xi|^{\beta}}
$$

where $\beta>0$ is the exponent parameter, $\omega>0$ is the scaling factor and $\Gamma$ is the gamma function. In the following, we will restrict to "heavy tail" log-concave distributions within this class by choosing $\beta \in[1,2]$. Within this class, $\beta=1$ corresponds to the Laplace distribution and $\beta=2$ to the Gaussian one. In addition, the differential entropy

A. Fraysse and B. Pesquet-Popescu are with the Ecole Nationale Supérieure des Télécommunications, Signal and Image Processing Department, 46 rue Barrault, 75634 Paris Cédex 13, France. E-mail: \{fraysse, pesquet\}@tsi.enst.fr.

J.-C. Pesquet is with the Institut Gaspard Monge and CNRS-UMR 8049, Université de Paris-Est Marne-la-Vallée, 77454 Marne-la-Vallée Cedex 2, France. E-mail: pesqueteuniv-mlv.fr. 
of this distribution [4] is: ${ }^{1}$

$$
h_{\beta}(\omega)=-\int_{-\infty}^{\infty} f(\xi) \ln f(\xi) d \xi=\ln \left(\frac{2 \Gamma(1 / \beta)}{\beta \omega^{1 / \beta}}\right)+\frac{1}{\beta} .
$$

When the data to be modelled become sparser - which may arise in particular when considering appropriate redundant frame decompositions of regular signals - an alternative Bernoulli-GG (BGG) model can be adopted. The BGG distribution is defined by:

$$
\forall \xi \in \mathbb{R}, \quad g(\xi)=(1-\epsilon) \delta(\xi)+\epsilon f(\xi)
$$

where $\epsilon \in[0,1]$ is the mixture parameter and $\delta$ denotes the Dirac distribution (i.e. point mass at 0 ). In particular, the Bernoulli-Gaussian model has been considered in several studies for modelling wavelet coefficients [5], [6].

The purpose of this paper is to study the discrete entropy of a BGG distributed memoryless source $X$ after a symmetric scalar uniform quantization [7]. More precisely, let $q>0$ be the quantizer step-size, the output $\bar{X}$ of the quantizer is given by:

$$
\bar{X}=r_{0}=0, \quad \text { if }|X|<\frac{q}{2},
$$

and, for all $i \in \mathbb{Z}$ such that $i \neq 0$,

$$
\bar{X}=r_{i}, \quad \text { if }\left(|i|-\frac{1}{2}\right) q \leq|X|<\left(|i|+\frac{1}{2}\right) q
$$

where the quantization levels are given by

$$
\forall i \geq 1, \quad r_{i}=-r_{-i}=(i+\zeta) q
$$

and $\zeta \in[-1 / 2,1 / 2]$ is an "offset" parameter, indicating the shift of the reconstruction level with respect to the middle of the quantization interval. Note that we will not consider any saturation effect. The most commonly used quantization rule corresponds to the case when $\zeta=0$ (i.e., mid-point reconstruction). For example, this rule constitutes the basic ingredient of many encoding strategies (e.g. embedded zero-trees [8], SPIHT [9], EZBC [10], EBCOT [11]...) which have been developed for wavelet-based image compression techniques.

The efficiency of uniform quantization at high bitrate was shown by Gish and Pierce [12] for power function costs. The performance of optimum scalar quantizers subject to an entropy constraint was investigated through numerical methods [13], [14] for various memoryless source probability densities (e.g. uniform, Gaussian, Laplacian, GG) at low resolution. In [15], a parametric form of the operational distortion-rate function of a scalar quantizer was derived for a uniformly distributed source and a wide class of difference distortion measures. In [16], an approach for designing entropy scalar constrained quantizers for exponential and Laplace distribution was proposed and comparisons were made with uniform quantizers. Recently in [17], the asymptotic behaviour of a uniform quantizer with centroid recontruction levels and an offset parameter was characterized at low resolution for a memoryless Gaussian source and a squared error distortion measure. In another useful reference [18], optimal transform coding of Gaussian vector scale mixtures, which include GG sources as particular cases, was studied at high bitrate, for

\footnotetext{
${ }^{1}$ For simplicity, the entropies will be expressed in Nats.
} 
quadratic criteria. Some studies, e.g. [19]-[22] have also considered the use of Laplace and GG probability models in modern compression systems. All the previously cited papers deal with sources having an absolutely continuous probability distribution. For small distortion, an asymptotic formula for the rate-distortion function of memoryless sources following a mixed probability distribution was given in [23]. These results were extended in [24] to a general class of vector sources.

The contributions of this paper are the following:

- Close approximations of the entropy of uniformly quantized GG and BGG sources are obtained. We provide order $n$ approximations of the entropy and characterize the approximation error. The asymptotic behaviour of the entropy is deduced. At high resolution we obtain a refinement of the well-known Bennett formula, and at low resolution a parallel with a discrete three-state source is drawn.

- Asymptotic expressions of the distortion at low and high resolutions are provided for an order $p(p \geq 1)$ moment error measure. Taking real values, the parameter $p$ provides flexibility in the choice of the distortion function, for example in order to better fit the Human Visual System (HVS). This can be more appropriate for designing distortion measures in the transform domain, as necessary in image/video coding systems, in which rate-distortion criteria often are evaluated based on coefficients, while aiming at minimizing the visual impact on the reconstructed image.

The introduction of such a real-valued exponent in the distortion measure also raises new issues for characterizing the optimality, depending on the source and quantizer features. More precisely, we show that the asymptotic expressions (for $q \rightarrow 0$ and $q \rightarrow \infty$ ) of the order $p$ measure depend, of course, on the parameters $\beta$ and $\epsilon$ of the BGG source, but they may take different forms depending on the values of $p$ and on the reconstruction offset. The expressions of the distortion at low and high bitrate for integer values $p=1$ (Mean Absolute Error) and $p=2$ (Mean Square Error) are found as particular cases.

- Accurate formulas for the corresponding operational rate-distortion functions are derived at high bitrate. The loss in performance w.r.t. the Shannon lower bound related to the use of a uniform scalar quantizer is evaluated as a function of $p$ and $\epsilon$.

- The slope factors of the operational rate-distortion functions at low bitrate are also determined. The dependence w.r.t. $p$ and $\beta$ is studied, in particular, and the relations with the Shannon optimal rate-distortion bound are examined. An important result which is shown, in particular, is that, at low resolution, the uniform quantizer is optimal for $\beta<p$, as soon as $\zeta<0$, while for $p=\beta$ the optimal reconstruction level is obtained for $\zeta=-1 / 2$. This result, in turn, allows us to derive the slope of the Shannon rate-distortion function in these cases.

The remainder of the paper is organized as follows: in Section II, we derive approximation formulas for calculating the entropy of a uniformly quantized GG source. These results are extended to quantized BGG sources in Section III. We derive these results through asymptotic expansions of the incomplete Gamma functions around 0 and around $\infty$. In Section IV, we provide asymptotic results for both high and low bitrates concerning the operational rate- 
distortion functions for the BGG distribution. An illustration of the application of these results to transform coding is given in Section V and some conclusive remarks are drawn in Section VI.

\section{ENTROPY OF QUANTIZED GG RANDOM VARIABLES}

Let us first assume that the random variable $X$ is distributed according to (1). The entropy of $\bar{X}$ is expressed as

$$
H_{f}(\bar{q})=-\sum_{i=-\infty}^{\infty} \mathrm{P}\left(\bar{X}=r_{i}\right) \ln \mathrm{P}\left(\bar{X}=r_{i}\right)
$$

where $\bar{q}=\omega^{1 / \beta} q$ is the normalized quantization step-size. Let, for all $a \in \mathbb{R}_{+}^{*}, Q_{a}$ be the normalized incomplete gamma function [25], defined by

$$
\forall \xi \in \mathbb{R}, \quad Q_{a}(\xi)=\frac{1}{\Gamma(a)} \int_{0}^{\xi} \theta^{a-1} e^{-\theta} d \theta .
$$

It is recalled that

$$
\begin{aligned}
\forall \xi \in \mathbb{R}, \quad & Q_{1}(\xi)=1-e^{-\xi} \\
& Q_{1 / 2}(\xi)=\operatorname{erf}(\sqrt{\xi})
\end{aligned}
$$

where $\operatorname{erf}(\cdot)$ is the error function. It follows that

$$
H_{f}(\bar{q})=-\mathrm{p}_{0} \ln \mathrm{p}_{0}-2 \sum_{i=1}^{\infty} \mathrm{p}_{i} \ln \mathrm{p}_{i}
$$

where the probability of the zero level is

$$
\mathrm{p}_{0}=2 \int_{0}^{\frac{q}{2}} f(\xi) d \xi=Q_{1 / \beta}\left(\bar{q}_{1}^{\beta}\right)
$$

and the probability of the $r_{i}$ reconstruction level is

$$
\mathrm{p}_{i}=\int_{\left(i-\frac{1}{2}\right) q}^{\left(i+\frac{1}{2}\right) q} f(\xi) d \xi=\frac{1}{2}\left(Q_{1 / \beta}\left(\bar{q}_{i+1}^{\beta}\right)-Q_{1 / \beta}\left(\bar{q}_{i}^{\beta}\right)\right), \quad i \geq 1,
$$

$\bar{q}_{i}=(i-1 / 2) \bar{q}$ being the $i$-th normalized decision level. In order to simplify the notations, the dependence of $\mathrm{p}_{i}$ on $\bar{q}$ will not be made explicit.

Example 1: For the Laplace distribution, by using (9), the following expression of the function $H_{f}$ is obtained:

$$
\begin{aligned}
H_{f}(\bar{q}) & =-\left(1-e^{-\frac{\bar{q}}{2}}\right) \ln \left(1-e^{-\frac{\bar{q}}{2}}\right)-e^{-\frac{\bar{q}}{2}} \ln \left(\frac{1-e^{-\bar{q}}}{2}\right)+\bar{q}\left(1-e^{-\bar{q}}\right) \sum_{i=1}^{\infty}\left(i-\frac{1}{2}\right) e^{-\left(i-\frac{1}{2}\right) \bar{q}} \\
& =-\left(1-e^{-\frac{\bar{q}}{2}}\right) \ln \left(1-e^{-\frac{\bar{q}}{2}}\right)-e^{-\frac{\bar{q}}{2}} \ln \left(\frac{1-e^{-\bar{q}}}{2}\right)+\bar{q} e^{-\frac{\bar{q}}{2}}\left(\frac{1}{2}+\frac{1}{e^{\bar{q}}-1}\right) .
\end{aligned}
$$

With the exception of the Laplace case, a simple expression of $H_{f}$ is not available. In order to get tractable approximations of $H_{f}$, the following result will be useful:

Lemma 1: For all $n \in \mathbb{N}$ with $n \geq 2$, we have

$$
0 \leq-\sum_{i=n}^{\infty} \mathrm{p}_{i} \ln \mathrm{p}_{i}+\int_{\left(n-\frac{1}{2}\right) q}^{\infty} f(\xi) \ln f(\xi) d \xi+\ln q \int_{\left(n-\frac{1}{2}\right) q}^{\infty} f(\xi) d \xi \leq \frac{\beta \bar{q}}{2 \Gamma(1 / \beta)}\left(\frac{2 n}{2 n-1}\right)^{\beta-1} e^{-\bar{q}_{n}^{\beta}} .
$$

Proof: See Appendix A. 
This lemma allows us to derive the following approximation formula for the discrete entropy of the quantized GG random variable:

Proposition 1: Let $n \geq 2$. We have

$$
H_{f}(\bar{q})=H_{f}^{(n)}(\bar{q})+\Delta_{n}
$$

where

$$
H_{f}^{(n)}(\bar{q})=-\mathrm{p}_{0} \ln \mathrm{p}_{0}-2 \sum_{i=1}^{n-1} \mathrm{p}_{i} \ln \mathrm{p}_{i}+\left(h_{\beta}(1)-\ln \bar{q}\right)\left(1-Q_{1 / \beta}\left(\bar{q}_{n}^{\beta}\right)\right)+\frac{\bar{q}_{n}}{\Gamma(1 / \beta)} e^{-\bar{q}_{n}^{\beta}}
$$

and

$$
0 \leq \Delta_{n} \leq \bar{\Delta}_{n}=\frac{\beta \bar{q}}{\Gamma(1 / \beta)}\left(\frac{2 n}{2 n-1}\right)^{\beta-1} e^{-\bar{q}_{n}^{\beta}} .
$$

Proof: For all $n \in \mathbb{N}^{*}$, we have

$$
2 \int_{\left(n-\frac{1}{2}\right) q}^{\infty} f(\xi) d \xi=1-Q_{1 / \beta}\left(\bar{q}_{n}^{\beta}\right)
$$

and

$$
\begin{aligned}
2 \int_{\left(n-\frac{1}{2}\right) q}^{\infty} f(\xi) \ln f(\xi) d \xi & =-2 \ln \left(\frac{2 \Gamma(1 / \beta)}{\beta \omega^{1 / \beta}}\right) \int_{\left(n-\frac{1}{2}\right) q}^{\infty} f(\xi) d \xi-2 \omega \int_{\left(n-\frac{1}{2}\right) q}^{\infty} \xi^{\beta} f(\xi) d \xi \\
& =-\ln \left(\frac{2 \Gamma(1 / \beta)}{\beta \omega^{1 / \beta}}\right)\left(1-Q_{1 / \beta}\left(\bar{q}_{n}^{\beta}\right)\right)-\frac{1}{\Gamma(1 / \beta)} \int_{\bar{q}_{n}^{\beta}}^{\infty} \theta^{1 / \beta} e^{-\theta} d \theta \\
& =\left(\ln \left(\omega^{1 / \beta}\right)-h_{\beta}(1)\right)\left(1-Q_{1 / \beta}\left(\bar{q}_{n}^{\beta}\right)\right)-\frac{\bar{q}_{n}}{\Gamma(1 / \beta)} e^{-\bar{q}_{n}^{\beta}}
\end{aligned}
$$

Then, the result straightforwardly follows from (11) and (15).

As illustrated by Fig. $1, H_{f}^{(2)}$ provides a tight lower approximation of $H_{f}$ whereas $H_{f}^{(3)}+\bar{\Delta}_{3}$ provides a good upper approximation of $H_{f}$. In addition, choosing $n=2$ is enough to predict both the high resolution and low resolution behaviour of the entropy of the quantized variable. This is summarized in the following statement:

Proposition 2: As $\bar{q} \rightarrow 0$, we have ${ }^{2}$

$$
H_{f}(\bar{q})=h_{\beta}(1)-\ln \bar{q}+O(\bar{q})
$$

As $\bar{q} \rightarrow \infty$, we have

$$
\begin{aligned}
H_{f}(\bar{q}) & =-\mathrm{p}_{0} \ln \mathrm{p}_{0}-\left(1-\mathrm{p}_{0}\right) \ln \left(\frac{1-\mathrm{p}_{0}}{2}\right)+O\left(\bar{q} e^{-(3 \bar{q} / 2)^{\beta}}\right) \\
& =\frac{\bar{q} e^{-\bar{q}^{\beta} / 2^{\beta}}}{2 \Gamma(1 / \beta) \tilde{q}^{\beta}}\left(1+\frac{1-1 / \beta}{\tilde{q}^{2 \beta}}\right)\left(\left(\frac{\bar{q}}{2}\right)^{\beta}+\ln \left(\frac{\tilde{q}^{\beta}}{\bar{q}}\right)+\ln (4 e \Gamma(1 / \beta))+O\left(\frac{1}{\bar{q}^{2 \beta}}\right)\right)
\end{aligned}
$$

where $\tilde{q}=\left(2^{-\beta} \bar{q}^{\beta}+1-1 / \beta\right)^{1 / \beta}$.

Proof: See Appendix B.

\section{Remark 1:}

(i) The obtained high resolution behaviour (when $\bar{q}$ is small) is well known [12]. It is sometimes referred to as Bennett's formula.

${ }^{2}$ Let $a: \mathbb{R}_{+}^{*} \rightarrow \mathbb{R}$, and $b: \mathbb{R}_{+}^{*} \rightarrow \mathbb{R}$, we use the notation $a(\xi)=O(b(\xi))$ as $\xi \rightarrow 0$ (resp. $\xi \rightarrow \infty$ ) to say that there exists $\eta>0$ such that $a(\xi) / b(\xi)$ is bounded for all $\xi \in(0, \eta)$ (resp. $\xi \in(\eta, \infty)$ ). In addition, $a(\xi)=o(b(\xi))$ as $\xi \rightarrow \xi_{0} \in[0, \infty]$ if $\lim _{\xi \rightarrow \xi_{0}} a(\xi) / b(\xi)=0$. 
(ii) In accordance with intuition, (22) shows that, at low resolution ( $\bar{q}$ large), the entropy is similar to that of a 3 -state discrete source with probability $\left(\frac{1-p_{0}}{2}, p_{0}, \frac{1-p_{0}}{2}\right)$. From Fig. 2, it can be observed that this results in a close approximation of the entropy for large enough values of the quantization step. The plots also show that the maximum value of the approximations given by (21) and (22) provides a reasonable lower approximation of the entropy.

(iii) As predicted by our calculations, Fig. 2 shows that, in general, (23) provides a less precise approximation of the entropy than (22) at low resolution. In addition, it can be observed that for a Laplace distribution (23) reduces to

$$
H_{f}(\bar{q})=e^{-\frac{\bar{q}}{2}}\left(\frac{\bar{q}}{2}+\ln 2+1+O\left(\frac{1}{\bar{q}^{2}}\right)\right) .
$$

More generally, for every $\beta \in[1,2]$, (23) allows us to state that, as $\bar{q} \rightarrow \infty$,

$$
H_{f}(\bar{q})=\frac{\bar{q} e^{-\bar{q}^{\beta} / 2^{\beta}}}{2 \Gamma(1 / \beta)}\left(1+O\left(\frac{\ln \bar{q}}{\bar{q}^{\beta}}\right)\right) .
$$

In the Gaussian case, this result is in agreement with [17, Theorem 6] (by setting the offset parameter in this theorem to $1 / 2)$.

(iv) It is clear that all the results in this section (as well as the ones in the next section) are independent of the values taken by the quantization levels. In particular, they are valid for quantization levels which are not constrained by (6), such as those corresponding to the centroids of the decision intervals for any distortion cost function.

\section{EXTENSION TO BGG RANDOM VARIABLES}

We now turn our attention to the more general mixture model given by (3). By using the following lemma, we will see that the results derived in the previous section are directly extendable.

Lemma 2: Let $X$ be a random variable distributed according to (3). The entropy of the associated uniformly quantized variable $\bar{X}$ is given by

$$
H_{g}(\epsilon, \bar{q})=\epsilon H_{f}(\bar{q})+\Phi\left(\mathrm{p}_{0}, \epsilon\right), \quad \epsilon \in[0,1]
$$

where $^{3}$

$$
\Phi\left(\mathrm{p}_{0}, \epsilon\right)=-\left(1-\epsilon\left(1-\mathrm{p}_{0}\right)\right) \ln \left(1-\epsilon\left(1-\mathrm{p}_{0}\right)\right)-\epsilon\left(1-\mathrm{p}_{0}\right) \ln \epsilon+\epsilon \mathrm{p}_{0} \ln \mathrm{p}_{0}
$$

In addition, $H_{g}(\cdot, \bar{q})$ is an increasing strictly concave function such that $H_{g}(0, \bar{q})=0$ and

$$
H_{g}(\epsilon, \bar{q})=\epsilon H_{f}(\bar{q})+(\epsilon-1) \ln \mathrm{p}_{0}+O\left((\epsilon-1)^{2}\right), \quad \text { as } \epsilon \rightarrow 1 .
$$

Proof: See Appendix C.

Approximation formulas for $H_{g}$ are straightforwardly derived from (26) by using (12) and (16)-(18). The following asymptotic results are also obtained:

\footnotetext{
${ }^{3}$ We adopt the convention $0 \ln 0=0$.
} 
Proposition 3: Let $\epsilon \in(0,1]$ be given. As $\bar{q} \rightarrow 0$, we have

$$
H_{g}(\epsilon, \bar{q})=H_{\epsilon}+\epsilon\left(h_{\beta}(1)-\ln \bar{q}+\mathbf{1}_{(0,1)}(\epsilon) \frac{\beta \bar{q}}{2 \Gamma(1 / \beta)} \ln \bar{q}\right)+O(\bar{q})
$$

where

$$
H_{\epsilon}=-\epsilon \ln \epsilon-(1-\epsilon) \ln (1-\epsilon)
$$

is the entropy of a Bernoulli variable with probability $(1-\epsilon, \epsilon)$ and $\mathbf{1}_{(0,1)}$ is the characteristic function of the interval $(0,1) \cdot{ }^{4}$ As $\bar{q} \rightarrow \infty$, we have

$$
\begin{aligned}
H_{g}(\epsilon, \bar{q}) & =-\left(1-\epsilon\left(1-\mathrm{p}_{0}\right)\right) \ln \left(1-\epsilon\left(1-\mathrm{p}_{0}\right)\right)-\epsilon\left(1-\mathrm{p}_{0}\right) \ln \left(\frac{\epsilon\left(1-\mathrm{p}_{0}\right)}{2}\right)+O\left(\bar{q} e^{-(3 \bar{q} / 2)^{\beta}}\right) \\
& =\frac{\epsilon \bar{q} e^{-\bar{q}^{\beta} / 2^{\beta}}}{2 \Gamma(1 / \beta) \tilde{q}^{\beta}}\left(1+\frac{1-1 / \beta}{\tilde{q}^{2 \beta}}\right)\left(\left(\frac{\bar{q}}{2}\right)^{\beta}+\ln \left(\frac{\tilde{q}^{\beta}}{\bar{q}}\right)+\ln \left(\frac{4 e \Gamma(1 / \beta)}{\epsilon}\right)+O\left(\frac{1}{\bar{q}^{2 \beta}}\right)\right) .
\end{aligned}
$$

Proof: According (12) and (93), as $\bar{q} \rightarrow 0$, we get:

$$
\Phi\left(\mathrm{p}_{0}, \epsilon\right)=-(1-\epsilon) \ln (1-\epsilon)-\epsilon \ln \epsilon+\epsilon \mathbf{1}_{(0,1)}(\epsilon) \frac{\beta \bar{q}}{2 \Gamma(1 / \beta)} \ln \bar{q}+O(\bar{q}) .
$$

Eq. (29) is then obtained by using (21) and (26).

As $\bar{q} \rightarrow \infty$, (31) readily follows from (26) and (22) whereas (32) is deduced from (101) and (104) similarly to the derivation of (23).

Remark 2:

(i) It is easy to show that $H_{g}(\epsilon, \bar{q})$ is a decreasing function of $\bar{q}$.

(ii) As a consequence of (29),

$$
H_{g}(\epsilon, \bar{q})=H_{\epsilon}+\epsilon\left(h_{\beta}(1)-\ln \bar{q}\right)+O(\bar{q} \ln \bar{q})
$$

So, the coding cost at high resolution becomes equivalent to that of coding separately a Bernoulli random variable with probability $(1-\epsilon, \epsilon)$ and a GG component occuring with probability $\epsilon$. We see that, when $\epsilon \neq 1$, the entropy remains, up to an error of order $\bar{q} \ln \bar{q}$, an affine function of $\ln \bar{q}$ as for the standard Bennett formula. However, the slope and the value at the origin of the graph of this function is modified and it is dependent on the value of the mixture parameter $\epsilon$. If we now consider a more precise approximation incuring an error of order $\bar{q}$ as in (21), a further additive term $\epsilon \beta \bar{q} \ln \bar{q} /(2 \Gamma(1 / \beta))$ has to be taken into account.

(iii) Eq. (31) shows that at low resolution the entropy is (up to a $O\left(\bar{q} e^{-(3 \bar{q} / 2)^{\beta}}\right)$ term) equal to that of a 3 -state discrete source with probability $\left(\epsilon \frac{1-\mathrm{p}_{0}}{2}, 1-\epsilon\left(1-\mathrm{p}_{0}\right), \epsilon \frac{1-\mathrm{p}_{0}}{2}\right)$.

(iv) An illustration of these results is shown in Fig. 3.

\section{ASYMPTOTIC RATE-DISTORTION RESUlTS}

We now examine some consequences of the results in the previous section in terms of rate-distortion theory. The distortion is here evaluated through the $p$-th order moment of the quantization error and is thus expressed as

$$
d_{p, \zeta}(\omega, \epsilon, q)=\mathrm{E}\left[|X-\bar{X}|^{p}\right]
$$

${ }^{4}$ This function is defined by: $\mathbf{1}_{(0,1)}(\epsilon)=1$ if $0<\epsilon<1,0$ otherwise. 
where $p \geq 1$ is a real exponent. In particular, $p=2$ corresponds to the mean square error criterion and $p=1$ to the mean absolute one. We will see that considering other values of $p$ may be of interest.

If $X$ is distributed according to (3), we have

$$
d_{p, \zeta}(\omega, \epsilon, q)=2 \epsilon\left(\int_{0}^{\frac{q}{2}} \xi^{p} f(\xi) d \xi+\sum_{i=1}^{\infty} \int_{\left(i-\frac{1}{2}\right) q}^{\left(i+\frac{1}{2}\right) q}\left|\xi-r_{i}\right|^{p} f(\xi) d \xi\right) .
$$

Let the normalized distortion be defined as

$$
\epsilon \bar{d}_{p, \zeta}(\bar{q})=\omega^{p / \beta} d_{p, \zeta}(\omega, \epsilon, q) .
$$

In the following, we will be mainly interested in the operational rate-distortion functions defined, for all $\bar{D} \geq 0$, by

$$
\forall \zeta \in[-1 / 2,1 / 2], \quad R_{p, \zeta}(\epsilon, \bar{D})=\inf _{\left\{\bar{q}>0 \mid \epsilon \bar{d}_{p, \zeta}(\bar{q}) \leq \bar{D}\right\}} H_{g}(\epsilon, \bar{q})
$$

and

$$
R_{p}(\epsilon, \bar{D})=\inf _{\zeta \in[-1 / 2,1 / 2]} R_{p, \zeta}(\epsilon, \bar{D}) .
$$

Notice that the above infimum can be calculated on a restricted interval as stated below:

Lemma 3: We have

$$
R_{p}(\epsilon, \bar{D})=\inf _{\zeta \in[-1 / 2,0]} R_{p, \zeta}(\epsilon, \bar{D})
$$

Proof: See Appendix D.

\section{A. High resolution behaviour}

Let us first look at the expression of the distortion at high resolution:

Lemma 4: Let $\nu=\left(\frac{1}{2}+\zeta\right)^{p+1}+\left(\frac{1}{2}-\zeta\right)^{p+1}$. As $\bar{q} \rightarrow 0$, we have

$$
\bar{d}_{p, \zeta}(\bar{q})=\frac{\nu \bar{q}^{p}}{p+1}(1+O(\bar{q})) .
$$

The above relation holds uniformly in $\zeta^{5}$

Proof: See Appendix E.

When $p=2$ and $\zeta=0$, (41) gives the classical formula for the mean square quantization error, at high resolution (see [26] for more details). This lemma allows us to derive the following rate-distortion result concerning the BGG model.

Proposition 4: For a random variable distributed according to (3) with $\epsilon \in(0,1]$, we have, as $\bar{D} \rightarrow 0$,

$$
R_{p, \zeta}(\epsilon, \bar{D})=H_{\epsilon}+\epsilon\left(h_{\beta}(1)-\frac{1}{p} \ln \left(\frac{(p+1) \bar{D}}{\epsilon \nu}\right)+\mathbf{1}_{(0,1)}(\epsilon)\left(\frac{(p+1) \bar{D}}{\epsilon \nu}\right)^{1 / p} \frac{\beta \ln \bar{D}}{2 p \Gamma(1 / \beta)}\right)+O\left(\bar{D}^{1 / p}\right)
$$

and

$$
R_{p}(\epsilon, \bar{D})=H_{\epsilon}+\epsilon\left(h_{\beta}(1)-\ln 2-\frac{1}{p} \ln \left(\frac{(p+1) \bar{D}}{\epsilon}\right)+\mathbf{1}_{(0,1)}(\epsilon)\left(\frac{(p+1) \bar{D}}{\epsilon}\right)^{1 / p} \frac{\beta \ln \bar{D}}{p \Gamma(1 / \beta)}\right)+O\left(\bar{D}^{1 / p}\right) .
$$

\footnotetext{
${ }^{5}$ This can be formally expressed as in (135).
} 
Proof: See Appendix F.

\section{Remark 3:}

(i) It can be noticed that, for a small distortion, the optimum operational rate-distortion performance is reached when $\nu=2^{-p}$, which corresponds to $\zeta=0$, that is the quantization reconstruction levels are the midpoints of the decision intervals.

(ii) If we omit the $O\left(\bar{D}^{1 / p} \ln \bar{D}\right)$ term, a loose approximation to (43) is given by

$$
R_{p}(\epsilon, \bar{D})=H_{\epsilon}+\epsilon\left(h_{\beta}(1)-\ln 2-\frac{1}{p} \ln \left(\frac{(p+1) \bar{D}}{\epsilon}\right)\right)+o(1) .
$$

This expression can be compared with the asymptotic form of the Shannon rate-distortion function [27]:

$$
\mathcal{R}_{p}(\epsilon, \bar{D})=\inf _{\left\{\widehat{X} \mid \mathrm{E}\left[|X-\widehat{X}|^{p}\right] \leq \omega-p / \beta \bar{D}\right\}} \mathcal{I}(X ; \widehat{X})
$$

where $\mathcal{I}(X ; \widehat{X})$ is the mutual information between the BGG random variable $X$ of interest and some arbitrary real-valued random variable $\widehat{X}$ defined on the same probability space. The asymptotic expression of $\mathcal{R}_{2}$ can be obtained from the results in [23], [24] (notice the correction brought by [24]). These results can be straightforwardly extended to any value of $p$ by using the fact that, subject to a $p$-th order moment upper bound, the differential entropy is maximized for a GG random variable of exponent $p$. Thus, we get

$$
\mathcal{R}_{p}(\epsilon, \bar{D})=H_{\epsilon}+\epsilon\left(h_{\beta}(1)-h_{p}(1)-\frac{1}{p} \ln \left(\frac{p \bar{D}}{\epsilon}\right)\right)+o(1) .
$$

Consequently, the performance loss related to the use of a uniform scalar quantizer is limited to

$$
\begin{aligned}
R_{p}(\epsilon, \bar{D})-\mathcal{R}_{p}(\epsilon, \bar{D}) & =\epsilon\left(h_{p}(1)-\ln 2-\frac{1}{p} \ln \left(\frac{1+p}{p}\right)\right)+o(1) \\
& =\epsilon\left(\ln \Gamma(1+1 / p)+\frac{1}{p}+\frac{1}{p} \ln \left(\frac{p}{1+p}\right)\right)+o(1)
\end{aligned}
$$

The difference is plotted in Fig. 4. In the case when $\epsilon=1$ and $p=2$, this gives the well-known difference of about 0.2546 bit [12].

\section{B. Low resolution behaviour}

Our main result in a low resolution context is the following:

Proposition 5: As $\bar{q} \rightarrow \infty$, we have

$$
\begin{aligned}
& \bar{d}_{p, \zeta}(\bar{q})= \\
& \begin{cases}\mu_{p}-\frac{\bar{q}^{p+1} e^{-\bar{q}^{\beta} / 2^{\beta}}}{2^{p+1} \Gamma(1 / \beta) \tilde{q}^{\beta}}\left(1-(1+2 \zeta)^{p}+\frac{p}{\beta \tilde{q}^{\beta}}\left(1+(1+2 \zeta)^{p-1}\right)+O\left(\frac{1}{\bar{q}^{2 \beta}}\right)\right) & \text { if } \zeta \neq-1 / 2 \text { or } p \geq 2 \\
\mu_{1}-\frac{\bar{q}^{2} e^{-\bar{q}^{\beta} / 2^{\beta}}}{4 \Gamma(1 / \beta) \tilde{q}^{\beta}}\left(1+O\left(\frac{1}{\bar{q}^{2 \beta}}\right)\right) & \text { if } \zeta=-1 / 2 \text { and } p=1 \\
\mu_{p}-\frac{\bar{q}^{p+1} e^{-\bar{q}^{\beta} / 2^{\beta}}}{2^{p+1} \Gamma(1 / \beta) \tilde{q}^{\beta}}\left(1+\frac{p}{\beta \tilde{q}^{\beta}}+O\left(\frac{1}{\bar{q}^{p \beta}}\right)\right) & \text { if } \zeta=-1 / 2 \text { and } 1<p<2\end{cases}
\end{aligned}
$$


where

$$
\mu_{p}=\omega^{p / \beta} \frac{\mathrm{E}\left[|X|^{p}\right]}{\epsilon}=\frac{\Gamma((p+1) / \beta)}{\Gamma(1 / \beta)} .
$$

Proof: For clarity, some of the technical parts of the proof have been deferred to Appendix G.

We start from the expression (164) of the distortion provided in Lemma 6 in Appendix G. We derive from (94) that

$$
\begin{aligned}
Q_{(p+1) / \beta}\left(\bar{q}_{1}^{\beta}\right) & =1-\frac{\bar{q}_{1}^{p+1} e^{-\bar{q}_{1}^{\beta}}}{\Gamma((p+1) / \beta)\left(\bar{q}_{1}^{\beta}-(p+1) / \beta+1\right)}\left(1+O\left(\frac{1}{\bar{q}^{2 \beta}}\right)\right) \\
& =1-\frac{\bar{q}_{1}^{p+1} e^{-\bar{q}_{1}^{\beta}}}{\Gamma((p+1) / \beta) \tilde{q}^{\beta}}\left(1+\frac{p}{\beta \tilde{q}^{\beta}}+O\left(\frac{1}{\bar{q}^{2 \beta}}\right)\right) .
\end{aligned}
$$

In addition,

$$
\beta \bar{q}_{1}^{-p-1+\beta} e^{\bar{q}_{1}^{\beta}} \int_{\bar{q}_{1}}^{\infty}\left|\xi-\bar{r}_{1}\right|^{p} e^{-\xi^{\beta}} d \xi=\int_{0}^{\infty}\left|\left(\frac{\theta}{\bar{q}_{1}^{\beta}}+1\right)^{1 / \beta}-2(\zeta+1)\right|^{p}\left(\frac{\theta}{\bar{q}_{1}^{\beta}}+1\right)^{1 / \beta-1} e^{-\theta} d \theta
$$

where the change of variable $\theta=\xi^{\beta}-\bar{q}_{1}^{\beta}$ has been made to obtain the last expression. By invoking now Lemma 7 in Appendix G (with $\alpha=\bar{q}_{1}^{-\beta}$ and $\lambda=2(1+\zeta)$ ), we get:

- when $\zeta \neq-1 / 2$,

$$
\beta \bar{q}_{1}^{-p-1+\beta} e^{\bar{q}_{1}^{\beta}} \int_{\bar{q}_{1}}^{\infty}\left|\xi-\bar{r}_{1}\right|^{p} e^{-\xi^{\beta}} d \xi=(1+2 \zeta)^{p}-(p+(\beta-1)(1+2 \zeta)) \frac{(1+2 \zeta)^{p-1}}{\beta \bar{q}_{1}^{\beta}}+O\left(\frac{1}{\bar{q}^{2 \beta}}\right)
$$

- when $\zeta=-1 / 2$ and $p=1$,

$$
\beta \bar{q}_{1}^{-2+\beta} e^{\bar{q}_{1}^{\beta}} \int_{\bar{q}_{1}}^{\infty}\left|\xi-\bar{r}_{1}\right| e^{-\xi^{\beta}} d \xi=\frac{1}{\beta \bar{q}_{1}^{\beta}}+O\left(\frac{1}{\bar{q}^{2 \beta}}\right)
$$

- when $\zeta=-1 / 2$ and $p>1$,

$$
\beta \bar{q}_{1}^{-p-1+\beta} e^{\bar{q}_{1}^{\beta}} \int_{\bar{q}_{1}}^{\infty}\left|\xi-\bar{r}_{1}\right|^{p} e^{-\xi^{\beta}} d \xi=O\left(\frac{1}{\bar{q}^{p \beta}}\right) .
$$

By using the relation $\bar{q}_{1}^{\beta}=\tilde{q}^{\beta}-1+1 / \beta$, we find in the first case that

$$
\beta \frac{\tilde{q}^{\beta}}{\bar{q}_{1}^{p+1}} e^{\bar{q}_{1}^{\beta}} \int_{\bar{q}_{1}}^{\infty}\left|\xi-\bar{r}_{1}\right|^{p} e^{-\xi^{\beta}} d \xi=(1+2 \zeta)^{p}-\frac{p(1+2 \zeta)^{p-1}}{\beta \tilde{q}^{\beta}}+O\left(\frac{1}{\bar{q}^{2 \beta}}\right)
$$

while, in the second one,

$$
\beta \frac{\tilde{q}^{\beta}}{\bar{q}_{1}^{2}} e^{\bar{q}_{1}^{\beta}} \int_{\bar{q}_{1}}^{\infty}\left|\xi-\bar{r}_{1}\right| e^{-\xi^{\beta}} d \xi=\frac{1}{\beta \tilde{q}^{\beta}}+O\left(\frac{1}{\bar{q}^{2 \beta}}\right)
$$

and in the third one,

$$
\beta \frac{\tilde{q}^{\beta}}{\bar{q}_{1}^{p+1}} e^{\bar{q}_{1}^{\beta}} \int_{\bar{q}_{1}}^{\infty}\left|\xi-\bar{r}_{1}\right|^{p} e^{-\xi^{\beta}} d \xi=O\left(\frac{1}{\bar{q}^{p \beta}}\right) .
$$

Altogether, (164), (50) and (55)-(57) yield (48), after some rearrangement.

\section{Remark 4:}

(i) An illustration of the results in Lemma 4 and Proposition 5 is displayed in Fig. 5. 
(ii) It may be interesting to note that for the standard quantization rule corresponding to $\zeta=0$, the zeroth order term in parentheses of (48) vanishes, so that this expression reduces to

$$
\bar{d}_{p, \zeta}(\bar{q})=\mu_{p}-\frac{p \bar{q}^{p+1} e^{-\bar{q}^{\beta} / 2^{\beta}}}{2^{p} \beta \Gamma(1 / \beta) \tilde{q}^{2 \beta}}\left(1+O\left(\frac{1}{\bar{q}^{\beta}}\right)\right)
$$

In contrast, when $\zeta \neq 0$, the zeroth order term is prevalent.

(iii) When $\zeta>0$ (resp. $\zeta \leq 0$ ), it can be observed that, for all $p \geq 1$, there exists $\tau_{p, \zeta}>0$ such that, if $\bar{q}>\tau_{p, \zeta}$ then $\bar{d}_{p, \zeta}(\bar{q})>\mu_{p}$ (resp. $\left.\bar{d}_{p, \zeta}(\bar{q})<\mu_{p}\right)$. This clearly shows that choosing $\zeta>0$ results in a poor quantization strategy.

(iv) According to (36) and (165), for all $\zeta \in[-1 / 2,1 / 2]$, a lower bound for $\bar{d}_{p, \zeta}(\bar{q})$ is

$$
\underline{d}_{p}(\bar{q})=2 \omega^{p / \beta} \int_{0}^{\frac{q}{2}} \xi^{p} f(\xi) d \xi=\mu_{p} Q_{(p+1) / \beta}\left(\frac{\bar{q}^{\beta}}{2^{\beta}}\right) .
$$

Basically, $\underline{d}_{p}$ is a measure of the truncation error for the values lying in the quantizer dead zone. By using (94), we get

$$
\underline{d}_{p}(\bar{q})=\mu_{p}-\frac{\bar{q}^{p+1-\beta} e^{-\bar{q}^{\beta} / 2^{\beta}}}{2^{p+1-\beta} \Gamma(1 / \beta)}\left(1+O\left(\frac{1}{\bar{q}^{\beta}}\right)\right) .
$$

It can be observed from Proposition 5 that $\bar{d}_{p, \zeta}$ is relatively close to this lower bound when $\zeta \neq 0$.

In order to derive rate-distortion results at low resolution, the following consequence of the previous proposition will be useful:

Corollary 1: For all $\epsilon \in(0,1]$, as $\bar{q} \rightarrow \infty$, we have:

- if $\zeta \neq 0$,

$$
\frac{H_{g}(\epsilon, \bar{q})}{\mu_{p}-\bar{d}_{p, \zeta}(\bar{q})}=\epsilon \frac{\bar{q}^{\beta-p}}{2^{\beta-p}\left(1-(1+2 \zeta)^{p}\right)}\left(1+O\left(\bar{q}^{-\beta}\left(1+\ln \bar{q}^{\beta-1}\right)\right)\right)
$$

- if $\zeta=0$,

$$
\frac{H_{g}(\epsilon, \bar{q})}{\mu_{p}-\bar{d}_{p, \zeta}(\bar{q})}=\epsilon \frac{\beta \bar{q}^{2 \beta-p}}{2^{2 \beta-p+1} p}\left(1+O\left(\bar{q}^{-\beta}\left(1+\ln \bar{q}^{\beta-1}\right)\right)\right) .
$$

Proof: Eq. (61) is deduced from (32) and (48). In turn, (32) and (58) lead to (62).

An interesting feature that allows us to quantify the low resolution behaviour is the slope of the tangent line to the operational rate-distortion curves at the extreme point corresponding to a zero rate and distortion $\epsilon \mu_{p}$. This one can be determined in an explicit manner as shown next.

Proposition 6: For all $\epsilon \in(0,1]$, if $\zeta<0$, we have ${ }^{6}$

$$
\lim _{\bar{D} \rightarrow\left(\epsilon \mu_{p}\right)^{-}} \frac{R_{p, \zeta}(\epsilon, \bar{D})}{\epsilon \mu_{p}-\bar{D}}= \begin{cases}\infty & \text { if } p<\beta \\ \left(1-(1+2 \zeta)^{p}\right)^{-1} & \text { if } p=\beta \\ 0 & \text { if } p>\beta\end{cases}
$$

${ }^{6}$ The notation $\bar{D} \rightarrow\left(\epsilon \mu_{p}\right)^{-}$means that $\bar{D}$ goes to $\epsilon \mu_{p}$ from below. 
and, if $\zeta=0$,

$$
\lim _{\bar{D} \rightarrow\left(\epsilon \mu_{p}\right)^{-}} \frac{R_{p, \zeta}(\epsilon, \bar{D})}{\epsilon \mu_{p}-\bar{D}}= \begin{cases}\infty & \text { if } p<2 \beta \\ \frac{1}{4} & \text { if } p=2 \beta \\ 0 & \text { if } p>2 \beta\end{cases}
$$

In addition,

$$
\lim _{\bar{D} \rightarrow\left(\epsilon \mu_{p}\right)^{-}} \frac{R_{p}(\epsilon, \bar{D})}{\epsilon \mu_{p}-\bar{D}}= \begin{cases}\infty & \text { if } p<\beta \\ 1 & \text { if } p=\beta \\ 0 & \text { if } p>\beta .\end{cases}
$$

Proof: See Appendix H.

The previous result allows us to deduce the left-sided derivative of the operational rate-distortion functions at $\epsilon \mu_{p}:$

Corollary 2: For all $\epsilon \in(0,1]$, if $\zeta<0$, we have

$$
\left.\frac{\partial R_{p, \zeta}(\epsilon, \bar{D})}{\partial \bar{D}}\right|_{\left(\epsilon \mu_{p}\right)^{-}}= \begin{cases}-\left(1-(1+2 \zeta)^{p}\right)^{-1} & \text { if } p=\beta \\ 0 & \text { if } p>\beta\end{cases}
$$

and, if $\zeta=0$,

$$
\left.\frac{\partial R_{p, \zeta}(\epsilon, \bar{D})}{\partial \bar{D}}\right|_{\left(\epsilon \mu_{p}\right)-}= \begin{cases}-\frac{1}{4} & \text { if } p=2 \beta \\ 0 & \text { if } p>2 \beta\end{cases}
$$

In addition, we have

$$
\left.\frac{\partial R_{p}(\epsilon, \bar{D})}{\partial \bar{D}}\right|_{\left(\epsilon \mu_{p}\right)^{-}}= \begin{cases}-1 & \text { if } p=\beta \\ 0 & \text { if } p>\beta\end{cases}
$$

Some final comments should be made about these results:

\section{Remark 5:}

(i) We see that the slope factor of the operational rate-distortion functions at low bitrate does not depend on $\epsilon$. However, the value of the distortion for which it is evaluated increases linearly w.r.t. $\epsilon$, that is when the data become less sparse.

(ii) When $p \geq \beta$, the slope factor of $R_{p}$ is equal to that of $\mathcal{R}_{p}$ (see (228) when $p=\beta$ and notice that the slope of $\mathcal{R}_{p}$ is nonpositive and it cannot be lower than that of $R_{p}$.) This shows the optimality at low resolution of uniform quantization for BGG random variables with exponent $\beta \leq p$.

(iii) When $p \leq 2 \beta$, Eq. (64) shows the suboptimality at low resolution of the quantizer corresponding to $\zeta=0$ (midpoint reconstruction levels), in spite of its frequent use in practical applications.

(iv) When $p=\beta$, the optimal rate-distortion performance is obtained asymptotically when $\zeta=-1 / 2$ (see Appendix $\mathrm{H})$, that is when $r_{i}=(i-1 / 2) q, i \geq 1$. The obtained low resolution rate-distortion behaviour is similar to that derived in [17]. The latter work is focussed on the Gaussian case $(\epsilon=1$ and $\beta=2)$ and the 
mean square criterion $(p=2)$ and the authors consider a uniform quantizer with optimal reconstruction levels in the mean square sense. To better highlight the connections with our results, it can be noticed that, when $p=2$ ( $\epsilon$ can be arbitrary), the centroids $\left(r_{i}^{*}\right)_{i \geq 1}$ of the positive decision intervals are explicitly given by:

$$
r_{i}^{*}=\frac{\int_{(i-1 / 2) q}^{(i+1 / 2) q} \xi f(\xi) d \xi}{\mathrm{p}_{i}}=\omega^{-1 / \beta} \frac{Q_{2 / \beta}\left(\bar{q}_{i+1}^{\beta}\right)-Q_{2 / \beta}\left(\bar{q}_{i}^{\beta}\right)}{Q_{1 / \beta}\left(\bar{q}_{i+1}^{\beta}\right)-Q_{1 / \beta}\left(\bar{q}_{i}^{\beta}\right)} .
$$

As $q \rightarrow \infty$, we deduce from (94) that

$$
r_{i}^{*}=(i-1 / 2) q\left(1+O\left(q^{-\beta}\right)\right)
$$

So, at low resolution, the centroids indeed converge to the lower bound of the decision intervals.

\section{Connections With TRAnsform Coding}

In typical transform coding applications, a vector of real-valued transform coefficients $\mathbf{X}=\left(X_{1}, \ldots, X_{n}\right)^{\top}$ is considered, the components of which can be modelled by BGG distributions. For simplicity, assume that these components are identically distributed, and that the vector of quantized values $\overline{\mathbf{X}}=\left(\bar{X}_{1}, \ldots, \bar{X}_{n}\right)^{\top}$ is obtained by applying the same uniform quantizer to each component of $\mathbf{X}$.

Our results concerning the entropy are directly applicable to this context since the entropy of $\overline{\mathbf{X}}$ is

$$
\mathbf{H}_{g}(\epsilon, \bar{q})=n H_{g}(\epsilon, \bar{q})
$$

where $H_{g}(\epsilon, \bar{q})$ is given by (26).

In turn, the expression of the distortion measure usually has to be modified since the reconstruction error depends on the reconstruction matrix $\boldsymbol{T}$ of size $m \times n$ (with $m \leq n$ ) and is given by $\boldsymbol{T}(\mathbf{X}-\overline{\mathbf{X}})$. Two simple cases are discussed next:

- In the case of a mean square error criterion, if $\boldsymbol{T}$ is orthogonal or if the components of the quantization error vector $\mathbf{X}-\overline{\mathbf{X}}$ are assumed uncorrelated (see [26] for a thorough study of the validity of this assumption), the distortion takes the form:

$$
\mathbf{d}_{2, \zeta}(\omega, \epsilon, q)=\frac{1}{n} \mathrm{E}\left[\|\boldsymbol{T}(\mathbf{X}-\overline{\mathbf{X}})\|^{2}\right]=\frac{\|\boldsymbol{T}\|_{\mathrm{F}}^{2}}{n} \mathrm{E}\left[\left(X_{1}-\bar{X}_{1}\right)^{2}\right]=\kappa d_{2, \zeta}(\omega, \epsilon, q)
$$

where $\|\cdot\|$ denotes the Euclidean norm, $\|\cdot\|_{F}$ is the Frobenius norm, $d_{2, \zeta}(\omega, \epsilon, q)$ is given by (36) and

$$
\kappa=\frac{1}{n}\|\boldsymbol{T}\|_{\mathrm{F}}^{2}
$$

- When $\boldsymbol{T}$ corresponds to a one-dimensional wavelet basis decomposition [1] performed up to a resolution level $J \geq 1$ and $n$ is multiple of $2^{J}$, the vector $\mathbf{X}$ can be rewritten as $\left[\left(X_{j, k}\right)_{1 \leq j \leq J, 0 \leq k<2^{-j} n}\left(X_{J+1, k}\right)_{0 \leq k<2^{-J} n}\right]^{\top}$ and a similar notation can be adopted for the vector $\overline{\mathbf{X}}$. Here, the components of $\mathbf{X}$ have been reindexed by introducing the resolution level $j \in\{1, \ldots, J\}$ and the time location $k$ where the detail coefficients $\left(X_{j, k}\right)_{1 \leq j \leq J, 0 \leq k<2^{-j} n}$ are defined. For simplicity, the approximation coefficients at resolution level $J$ have 
been denoted by $\left(X_{J+1, k}\right)_{0 \leq k<2^{-J} n}$. A useful norm in the context of wavelet representations is the Besov norm $\|\cdot\|_{B_{s, p, p^{\prime}}}$ with $s>0$ and $\left(p, p^{\prime}\right) \in\left[1, \infty\left[^{2}\right.\right.$ which applied to the reconstruction error yields:

$$
\begin{aligned}
\|\boldsymbol{T}(\mathbf{X}-\overline{\mathbf{X}})\|_{B_{s, p, p^{\prime}}}= & \left(\sum_{k=0}^{2^{-J} n-1}\left|X_{J+1, k}-\bar{X}_{J+1, k}\right|^{p}\right)^{1 / p} \\
& +\left(\sum_{j=1}^{J} 2^{-j p^{\prime}(s+1 / 2-1 / p)}\left(\sum_{k=0}^{2^{-j} n-1}\left|X_{j, k}-\bar{X}_{j, k}\right|^{p}\right)^{p^{\prime} / p}\right)^{1 / p^{\prime}} .
\end{aligned}
$$

More details concerning the relations between this norm and regular function spaces can be found in [28]. If we assume that the components of $\mathbf{X}-\overline{\mathbf{X}}$ are the first $n$ terms of an independent identically distributed sequence, the strong law of large number allows us to see that, as $n \rightarrow \infty$,

$$
\begin{array}{r}
\frac{1}{2^{-J} n} \sum_{k=0}^{2^{-J} n-1}\left|X_{J+1, k}-\bar{X}_{J+1, k}\right|^{p} \stackrel{\text { a.s. }}{\rightarrow} \mathrm{E}\left[\left|X_{J+1,0}-\bar{X}_{J+1,0}\right|^{p}\right] \\
\forall j \in\{1, \ldots, J\}, \quad \frac{1}{2^{-j} n} \sum_{k=0}^{2^{-j} n-1}\left|X_{j, k}-\bar{X}_{j, k}\right|^{p} \stackrel{\text { a.s. }}{\rightarrow} \mathrm{E}\left[\left|X_{j, 0}-\bar{X}_{j, 0}\right|^{p}\right]
\end{array}
$$

where $\stackrel{\text { a.s. }}{\rightarrow}$ denotes the almost sure convergence. Hence,

$$
\frac{1}{n}\|\boldsymbol{T}(\mathbf{X}-\overline{\mathbf{X}})\|_{B_{s, p, p^{\prime}}}^{p} \stackrel{\text { a.s. }}{\rightarrow} \kappa \mathrm{E}\left[\left|X_{1,0}-\bar{X}_{1,0}\right|^{p}\right]
$$

where

$$
\kappa=2^{-J / p}+2^{-(s+1 / 2)}\left(\frac{1-2^{-J p^{\prime}(s+1 / 2)}}{1-2^{-p^{\prime}(s+1 / 2)}}\right)^{1 / p^{\prime}} .
$$

This implies that, as a final form of the distortion function, one can take

$$
\mathbf{d}_{p, \zeta}(\omega, \epsilon, q)=\lim _{n \rightarrow \infty} \frac{1}{n} \mathrm{E}\left[\|\boldsymbol{T}(\mathbf{X}-\overline{\mathbf{X}})\|_{B_{s, p, p^{\prime}}}^{p}\right]=\kappa \mathrm{E}\left[\left|X_{1,0}-\bar{X}_{1,0}\right|^{p}\right]=\kappa d_{p, \zeta}(\omega, \epsilon, q) .
$$

Similar relations hold in the two-dimensional case [29].

So, compared with the distortion we considered in Section IV, just a scaling factor $\kappa$ has to be introduced in the two previous cases. In particular, by redefining the operational rate-distortion function $R_{p, \zeta}$ as: for all $\bar{D} \geq 0$,

$$
\forall \zeta \in[-1 / 2,1 / 2], \quad n R_{p, \zeta}(\epsilon, \bar{D})=\inf _{\left\{\bar{q}>0 \mid \omega^{p / \beta} \mathbf{d}_{p, \zeta}(\omega, \epsilon, q) \leq \kappa \bar{D}\right\}} \mathbf{H}_{g}(\epsilon, \bar{q})
$$

and, subsequently applying Definition (39) for $R_{p}$, the results in Propositions 4 and 6 and Corollary 2 are unchanged.

\section{CONCLUSION}

At high bitrate, the main result in this paper is Proposition 4 which provides accurate approximations of the operational rate-distortion functions for a uniformly quantized BGG source. At low bitrate, asymptotic formulas have also been obtained for the entropy in (32) and for the distortion in (48), which have allowed us in Proposition 6 to deduce the slope factor of the operational rate-distortion function for a distortion equal to $\epsilon \mu_{p}$. At high bitrate, the uniform quantizer remains suboptimal but the difference w.r.t. the Shannon optimal bound is a linear function of the mixture parameter $\epsilon$ (see (47)) and it therefore becomes smaller when the data are sparser. At low bitrate, 
we have generalized the results in [17] by showing that the slope factor of the rate-distortion function $R_{p}$ is the optimal one, provided that the order $p$ of the distortion measure is greater than or equal to the exponent $\beta$ and the positive (resp. negative) quantizer reconstruction levels are chosen equal to the lower (resp. upper) bounds of the decision intervals. The application of these results to transform coding was also briefly discussed in two simple cases.

\section{APPENDIX A}

Proof OF LEMMA 1

Since $f$ is a decreasing function on $\mathbb{R}_{+}$, we have, for all $i>0$,

$$
q f((i+1 / 2) q) \leq \mathrm{p}_{i} \leq q f((i-1 / 2) q)
$$

Noticing that

$$
-\mathrm{p}_{i} \ln \mathrm{p}_{i}+\int_{\left(i-\frac{1}{2}\right) q}^{\left(i+\frac{1}{2}\right) q} f(\xi) \ln f(\xi) d \xi=\int_{\left(i-\frac{1}{2}\right) q}^{\left(i+\frac{1}{2}\right) q} f(\xi)\left(\ln f(\xi)-\ln \mathrm{p}_{i}\right) d \xi
$$

we get the following inequality:

$$
-\mathrm{p}_{i} \ln \mathrm{p}_{i}+\int_{\left(i-\frac{1}{2}\right) q}^{\left(i+\frac{1}{2}\right) q} f(\xi) \ln f(\xi) d \xi \leq \int_{\left(i-\frac{1}{2}\right) q}^{\left(i+\frac{1}{2}\right) q} f(\xi)(\ln f(\xi)-\ln f((i+1 / 2) q)-\ln q) d \xi .
$$

On the other hand, from the positivity of the Kullback-Leibler divergence, we obtain

$$
\int_{\left(i-\frac{1}{2}\right) q}^{\left(i+\frac{1}{2}\right) q} \frac{f(\xi)}{\mathrm{p}_{i}} \ln \left(\frac{f(\xi) / \mathrm{p}_{i}}{1 / q}\right) d \xi \geq 0
$$

which is equivalent to

$$
-\mathrm{p}_{i} \ln \mathrm{p}_{i}+\int_{\left(i-\frac{1}{2}\right) q}^{\left(i+\frac{1}{2}\right) q} f(\xi) \ln f(\xi) d \xi+\ln q \int_{\left(i-\frac{1}{2}\right) q}^{\left(i+\frac{1}{2}\right) q} f(\xi) d \xi \geq 0 .
$$

Therefore, when $i \geq 2$, (83) and (85) yield

$$
\begin{aligned}
0 \leq-\mathrm{p}_{i} & \ln \mathrm{p}_{i}+\int_{\left(i-\frac{1}{2}\right) q}^{\left(i+\frac{1}{2}\right) q} f(\xi) \ln f(\xi) d \xi+\ln q \int_{\left(i-\frac{1}{2}\right) q}^{\left(i+\frac{1}{2}\right) q} f(\xi) d \xi \\
\leq & (\ln f((i-1 / 2) q)-\ln f((i+1 / 2) q)) \int_{\left(i-\frac{1}{2}\right) q}^{\left(i+\frac{1}{2}\right) q} f(\xi) d \xi \\
& =\omega q^{\beta}\left((i+1 / 2)^{\beta}-(i-1 / 2)^{\beta}\right) \int_{\left(i-\frac{1}{2}\right) q}^{\left(i+\frac{1}{2}\right) q} f(\xi) d \xi .
\end{aligned}
$$

Besides,

$$
\begin{aligned}
(i+1 / 2)^{\beta}-(i-1 / 2)^{\beta} & =i^{\beta}\left(\left(1+\frac{1}{2 i}\right)^{\beta}-\left(1-\frac{1}{2 i}\right)^{\beta}\right) \\
& =2 i^{\beta} \sum_{k=0}^{\infty} \frac{\beta(\beta-1) \ldots(\beta-2 k)}{(2 k+1) !}\left(\frac{1}{2 i}\right)^{2 k+1} \leq \beta i^{\beta-1}
\end{aligned}
$$

where the upper bound follows from the fact that $1 \leq \beta \leq 2$. Consequently,

$$
0 \leq-\mathrm{p}_{i} \ln \mathrm{p}_{i}+\int_{\left(i-\frac{1}{2}\right) q}^{\left(i+\frac{1}{2}\right) q} f(\xi) \ln f(\xi) d \xi+\ln q \int_{\left(i-\frac{1}{2}\right) q}^{\left(i+\frac{1}{2}\right) q} f(\xi) d \xi \leq \beta \omega q \int_{\left(i-\frac{1}{2}\right) q}^{\left(i+\frac{1}{2}\right) q}(\xi+q / 2)^{\beta-1} f(\xi) d \xi .
$$


It can be deduced that, for $n \geq 2$,

$$
0 \leq-\sum_{i=n}^{\infty} \mathrm{p}_{i} \ln \mathrm{p}_{i}+\int_{\left(n-\frac{1}{2}\right) q}^{\infty} f(\xi) \ln f(\xi) d \xi+\ln q \int_{\left(n-\frac{1}{2}\right) q}^{\infty} f(\xi) d \xi \leq I
$$

where

$$
I=\beta \omega q \int_{\left(n-\frac{1}{2}\right) q}^{\infty}(\xi+q / 2)^{\beta-1} f(\xi) d \xi .
$$

By noticing that $\xi \geq(n-1 / 2) q \Leftrightarrow \xi+q / 2 \leq 2 n \xi /(2 n-1)$, it can be concluded that

$$
I \leq \beta \omega q\left(\frac{2 n}{2 n-1}\right)^{\beta-1} \int_{\left(n-\frac{1}{2}\right) q}^{\infty} \xi^{\beta-1} f(\xi) d \xi=\frac{\beta \bar{q}}{2 \Gamma(1 / \beta)}\left(\frac{2 n}{2 n-1}\right)^{\beta-1} e^{-\left(n-\frac{1}{2}\right)^{\beta} \bar{q}^{\beta}} .
$$

Combining (89) and (91) leads to (15).

\section{APPENDIX B}

\section{Proof of Proposition 2}

Setting $n=2$ in (16) and (17), we get

$$
\begin{aligned}
H_{f}(\bar{q})= & -Q_{1 / \beta}\left(\bar{q}_{1}^{\beta}\right) \ln Q_{1 / \beta}\left(\bar{q}_{1}^{\beta}\right)-\left(Q_{1 / \beta}\left(\bar{q}_{2}^{\beta}\right)-Q_{1 / \beta}\left(\bar{q}_{1}^{\beta}\right)\right) \ln \left(\frac{Q_{1 / \beta}\left(\bar{q}_{2}^{\beta}\right)-Q_{1 / \beta}\left(\bar{q}_{1}^{\beta}\right)}{2}\right) \\
& +\left(h_{\beta}(1)-\ln \bar{q}\right)\left(1-Q_{1 / \beta}\left(\bar{q}_{2}^{\beta}\right)\right)+\frac{\bar{q}_{2}}{\Gamma(1 / \beta)} e^{-\bar{q}_{2}^{\beta}}+\Delta_{2} .
\end{aligned}
$$

We further know [30, p.891] that, for all $a \leq 1$,

$$
Q_{a}(\xi)=\frac{\xi^{a}}{a \Gamma(a)}+O\left(\xi^{2 a}\right), \quad \text { as } \xi \rightarrow 0
$$

and [25] for all $a>0$,

$$
Q_{a}(\xi)=1-\frac{\xi^{a} e^{-\xi}}{\Gamma(a)(\xi-a+1)}\left(1+\frac{1-a}{(\xi-a+1)^{2}}+O\left(\xi^{-3}\right)\right), \quad \text { as } \xi \rightarrow \infty .
$$

When $\bar{q} \rightarrow 0$, (18) allows us to show that

$$
\Delta_{2}=O(\bar{q})
$$

and, by using (92) and (93), we obtain

$$
\begin{aligned}
H_{f}(\bar{q})= & -\frac{\beta \bar{q}_{1}}{\Gamma(1 / \beta)}\left(\ln \bar{q}+\ln \left(\frac{\beta}{2 \Gamma(1 / \beta)}\right)\right)+O\left(\bar{q}^{2} \ln \bar{q}\right) \\
& -\frac{\beta \bar{q}}{\Gamma(1 / \beta)}\left(\ln \bar{q}+\ln \left(\frac{\beta}{2 \Gamma(1 / \beta)}\right)\right)+O\left(\bar{q}^{2} \ln \bar{q}\right)+\left(h_{\beta}(1)-\ln \bar{q}\right)\left(1-\frac{\beta \bar{q}_{2}}{\Gamma(1 / \beta)}+O\left(\bar{q}^{2}\right)\right)+O(\bar{q})
\end{aligned}
$$

which leads to (21).

When $\bar{q} \rightarrow \infty$, we deduce from (94) that

$$
Q_{1 / \beta}\left(\bar{q}_{2}^{\beta}\right)-Q_{1 / \beta}\left(\bar{q}_{1}^{\beta}\right)=1-Q_{1 / \beta}\left(\bar{q}_{1}^{\beta}\right)+O\left(\bar{q}^{1-\beta} e^{-\bar{q}_{2}^{\beta}}\right) .
$$

Invoking again (94), we have

$$
\ln \left(\frac{Q_{1 / \beta}\left(\bar{q}_{2}^{\beta}\right)-Q_{1 / \beta}\left(\bar{q}_{1}^{\beta}\right)}{2}\right)=\ln \left(\frac{1-Q_{1 / \beta}\left(\bar{q}_{1}^{\beta}\right)}{2}\right)+O\left(e^{\bar{q}_{1}^{\beta}-\bar{q}_{2}^{\beta}}\right)
$$


and

$$
\begin{array}{r}
-\left(Q_{1 / \beta}\left(\bar{q}_{2}^{\beta}\right)-Q_{1 / \beta}\left(\bar{q}_{1}^{\beta}\right)\right) \ln \left(\frac{Q_{1 / \beta}\left(\bar{q}_{2}^{\beta}\right)-Q_{1 / \beta}\left(\bar{q}_{1}^{\beta}\right)}{2}\right)=-\left(1-Q_{1 / \beta}\left(\bar{q}_{1}^{\beta}\right)\right) \ln \left(\frac{1-Q_{1 / \beta}\left(\bar{q}_{1}^{\beta}\right)}{2}\right) \\
+O\left(\bar{q} e^{-\bar{q}_{2}^{\beta}}\right)
\end{array}
$$

where the fact that $\ln \left(Q_{1 / \beta}\left(\bar{q}_{2}^{\beta}\right)-Q_{1 / \beta}\left(\bar{q}_{1}^{\beta}\right)\right)=O\left(\bar{q}^{\beta}\right)$ has been used. Now, from (12), (18) and (92), we get

$$
H_{f}(\bar{q})=-\mathrm{p}_{0} \ln \mathrm{p}_{0}-\left(1-\mathrm{p}_{0}\right) \ln \left(\frac{1-\mathrm{p}_{0}}{2}\right)+\left(h_{\beta}(1)-\ln \bar{q}\right) \frac{\bar{q}_{2}^{1-\beta}}{\Gamma(1 / \beta)} e^{-\bar{q}_{2}^{\beta}}+O\left(\bar{q} e^{-\bar{q}_{2}^{\beta}}\right)
$$

from which (22) follows. Using now (12) and (94), we find that

$$
\mathrm{p}_{0}=1-\frac{\bar{q}_{1} e^{-\bar{q}_{1}^{\beta}}}{\Gamma(1 / \beta)\left(\bar{q}_{1}^{\beta}+1-1 / \beta\right)}\left(1+\frac{1-1 / \beta}{\left(\bar{q}_{1}^{\beta}+1-1 / \beta\right)^{2}}+O\left(\frac{1}{\bar{q}^{3 \beta}}\right)\right)
$$

and, consequently,

$$
\begin{aligned}
-\mathrm{p}_{0} \ln \mathrm{p}_{0} & =\frac{\bar{q}_{1} e^{-\bar{q}_{1}^{\beta}}}{\Gamma(1 / \beta)\left(\bar{q}_{1}^{\beta}+1-1 / \beta\right)}\left(1+\frac{1-1 / \beta}{\left(\bar{q}_{1}^{\beta}+1-1 / \beta\right)^{2}}+O\left(\frac{1}{\bar{q}^{3 \beta}}\right)\right) \\
\ln \left(\frac{1-\mathrm{p}_{0}}{2}\right) & =-\bar{q}_{1}^{\beta}-\ln \left(\bar{q}_{1}^{\beta}+1-1 / \beta\right)+\ln \bar{q}_{1}-\ln (2 \Gamma(1 / \beta))+O\left(\frac{1}{\bar{q}^{2 \beta}}\right) \\
-\left(1-\mathrm{p}_{0}\right) \ln \left(\frac{1-\mathrm{p}_{0}}{2}\right)= & \frac{\bar{q}_{1} e^{-\bar{q}_{1}^{\beta}}}{\Gamma(1 / \beta)\left(\bar{q}_{1}^{\beta}+1-1 / \beta\right)}\left(1+\frac{1-1 / \beta}{\left(\bar{q}_{1}^{\beta}+1-1 / \beta\right)^{2}}\right)\left(\bar{q}_{1}^{\beta}+\ln \left(\bar{q}_{1}^{\beta}+1-1 / \beta\right)\right. \\
& \left.-\ln \bar{q}_{1}+\ln (2 \Gamma(1 / \beta))\right)+O\left(\bar{q}^{1-3 \beta} e^{-\bar{q}_{1}^{\beta}}\right) .
\end{aligned}
$$

Eqs. (102) and (104) readily yield (23).

\section{APPENDIX C}

PROOF OF LEMMA 2

The entropy of the quantized BGG random variable is

$$
H_{g}(\epsilon, \bar{q})=-\mathrm{p}_{0}^{\prime} \ln \mathrm{p}_{0}^{\prime}-2 \sum_{i=1}^{\infty} \mathrm{p}_{i}^{\prime} \ln \mathrm{p}_{i}^{\prime}
$$

where

$$
\begin{aligned}
& \mathrm{p}_{0}^{\prime}=1-\epsilon+\epsilon \mathrm{p}_{0} \\
& \mathrm{p}_{i}^{\prime}=\epsilon \mathrm{p}_{i}, \quad i \geq 1 .
\end{aligned}
$$

This leads to

$$
\begin{aligned}
H_{g}(\epsilon, \bar{q}) & =-\left(1-\epsilon+\epsilon \mathrm{p}_{0}\right) \ln \left(1-\epsilon+\epsilon \mathrm{p}_{0}\right)-2 \epsilon\left(\sum_{i=1}^{\infty} \mathrm{p}_{i} \ln \mathrm{p}_{i}+\ln \epsilon \sum_{i=1}^{\infty} \mathrm{p}_{i}\right) \\
& =-\left(1-\epsilon+\epsilon \mathrm{p}_{0}\right) \ln \left(1-\epsilon+\epsilon \mathrm{p}_{0}\right)+\epsilon\left(H_{f}(\bar{q})+\mathrm{p}_{0} \ln \mathrm{p}_{0}\right)-\left(1-\mathrm{p}_{0}\right) \epsilon \ln \epsilon
\end{aligned}
$$


which yields (26).

In addition, we have, for all $\epsilon \in(0,1],^{7}$

$$
\begin{aligned}
\frac{\partial \Phi}{\partial \epsilon}\left(\mathrm{p}_{0}, \epsilon\right) & =\left(1-\mathrm{p}_{0}\right) \ln \left(\frac{1-\left(1-\mathrm{p}_{0}\right) \epsilon}{\epsilon}\right)+\mathrm{p}_{0} \ln \mathrm{p}_{0} \\
\frac{\partial^{2} \Phi}{\partial \epsilon^{2}}\left(\mathrm{p}_{0}, \epsilon\right) & =-\frac{\left(1-\mathrm{p}_{0}\right)^{2}}{1-\left(1-\mathrm{p}_{0}\right) \epsilon}-\frac{1-\mathrm{p}_{0}}{\epsilon}<0 .
\end{aligned}
$$

Therefore, $\epsilon \mapsto \Phi\left(\mathrm{p}_{0}, \epsilon\right)$ is a strictly concave function such that $\Phi\left(\mathrm{p}_{0}, 0\right)=0$ and

$$
\Phi\left(\mathrm{p}_{0}, \epsilon\right)=(\epsilon-1) \ln \mathrm{p}_{0}+O\left((\epsilon-1)^{2}\right), \quad \text { as } \epsilon \rightarrow 1 .
$$

Since $H_{g}(\cdot, \bar{q})$ is a linear perturbation of this function, it is also a strictly concave function with the desired values when $\epsilon=0$ or $\epsilon$ is close to 1 . To show that $H_{g}(\cdot, \bar{q})$ is increasing, it is then sufficient to check that its left-sided derivative at 1 is positive. According to (109), this derivative is given by

$$
\frac{\partial H_{g}}{\partial \epsilon}(1, \bar{q})=\ln \mathrm{p}_{0}+H_{f}(\bar{q}) .
$$

On the other hand, since $f$ is even and decreasing over $\mathbb{R}_{+}$,

$$
\forall i \geq 1, \quad \mathrm{p}_{i}<\mathrm{p}_{0} .
$$

Thus, according to (11),

$$
H_{f}(\bar{q})>-\mathrm{p}_{0} \ln \mathrm{p}_{0}-2 \ln \mathrm{p}_{0} \sum_{i=1}^{\infty} \mathrm{p}_{i}=-\ln \mathrm{p}_{0}
$$

that is, $\frac{\partial H_{g}}{\partial \epsilon}(1, \bar{q})>0$.

\section{APPENDIX D}

PROOF OF LEMMA 3

For every $i \geq 1$, the function

$$
\varphi_{p}: \mathbb{R}_{+} \rightarrow \mathbb{R}_{+}: r \mapsto \int_{(i-1 / 2) q}^{(i+1 / 2) q}|\xi-r|^{p} f(\xi) d \xi
$$

is convex on $[0, \infty)$ and its minimizer $r_{i}^{*} \in[(i-1 / 2) q,(i+1 / 2) q]$ satisfies

$$
\int_{(i-1 / 2) q}^{r_{i}^{*}}\left(r_{i}^{*}-\xi\right)^{p-1} f(\xi) d \xi=\int_{r_{i}^{*}}^{(i+1 / 2) q}\left(\xi-r_{i}^{*}\right)^{p-1} f(\xi) d \xi .
$$

(This minimizer is unique since, for $p>1, \varphi_{p}$ is strictly convex and, when $p=1, r_{i}^{*}$ is the median of $f$ over $[(i-1 / 2) q,(i+1 / 2) q]$, which is uniquely defined as $f$ is decreasing.) This minimizer belongs to $[(i-1 / 2) q, i q]$. Indeed, if $i q<r_{i}^{*} \leq(i+1 / 2) q \Leftrightarrow(i-1 / 2) q<2 r_{i}^{*}-(i+1 / 2) q \leq r_{i}^{*}$, we would have

$$
\begin{aligned}
0<\int_{(i-1 / 2) q}^{2 r_{i}^{*}-(i+1 / 2) q}\left(r_{i}^{*}-\xi\right)^{p-1} f(\xi) d \xi & =-\int_{2 r_{i}^{*}-(i+1 / 2) q}^{r_{i}^{*}}\left(r_{i}^{*}-\xi\right)^{p-1} f(\xi) d \xi+\int_{r_{i}^{*}}^{(i+1 / 2) q}\left(\xi-r_{i}^{*}\right)^{p-1} f(\xi) d \xi \\
& =\int_{r_{i}^{*}}^{(i+1 / 2) q}\left(\xi-r_{i}^{*}\right)^{p-1}\left(f(\xi)-f\left(2 r_{i}^{*}-\xi\right)\right) d \xi \leq 0
\end{aligned}
$$

\footnotetext{
${ }^{7}$ When $\epsilon=1$, the provided expressions correspond to left-sided derivatives.
} 
where the last inequality comes from the fact that $f$ is decreasing on $\mathbb{R}_{+}$.

The convexity of $\varphi_{p}$ implies that this function is increasing on $\left[r_{i}^{*}, \infty\right)$ and, since $r_{i}^{*} \leq i q$, we have, for every $r \in[i q,(i+1 / 2) q]$ :

$$
\int_{(i-1 / 2) q}^{(i+1 / 2) q}|\xi-i q|^{p} f(\xi) d \xi=\varphi_{p}(i q) \leq \varphi_{p}(r)=\int_{(i-1 / 2) q}^{(i+1 / 2) q}|\xi-r|^{p} f(\xi) d \xi .
$$

Recalling (36) and (37), we deduce that, for every $\zeta \in[0,1 / 2]$ and $\bar{q}>0$,

$$
\bar{d}_{p, 0}(\bar{q}) \leq \bar{d}_{p, \zeta}(\bar{q}) .
$$

Consequently, for every $\zeta \in[0,1 / 2]$ and $\bar{D}>0$,

$$
\left\{\bar{q}>0 \mid \bar{d}_{p, \zeta}(\bar{q}) \leq \bar{D}\right\} \subset\left\{\bar{q}>0 \mid \bar{d}_{p, 0}(\bar{q}) \leq \bar{D}\right\}
$$

which, according to (38), yields

$$
R_{p, \zeta}(\epsilon, \bar{D}) \geq R_{p, 0}(\epsilon, \bar{D})
$$

This shows that the infimum in (39) can be restricted to $\zeta \in[-1 / 2,0]$.

\section{APPENDIX E}

PROOF OF LEMMA 4

We have

$$
\bar{d}_{p, \zeta}(\bar{q})=2\left(\int_{0}^{\bar{q}_{1}} \xi^{p} f_{1}(\xi) d \xi+\sum_{i=1}^{\infty} \int_{\bar{q}_{i}}^{\bar{q}_{i+1}}\left|\xi-\bar{r}_{i}\right|^{p} f_{1}(\xi) d \xi\right)
$$

where $f_{1}(\xi)=\omega^{-1 / \beta} f\left(\omega^{-1 / \beta} \xi\right)$ and $\bar{r}_{i}=\omega^{1 / \beta} r_{i}$. Since $f_{1}$ is a decreasing function over $\mathbb{R}_{+}$, we first notice that

$$
0 \leq \int_{0}^{\bar{q}_{1}} \xi^{p} f_{1}(\xi) d \xi \leq f_{1}(0) \int_{0}^{\bar{q}_{1}} \xi^{p} d \xi=f_{1}(0) \frac{\bar{q}_{1}^{p+1}}{p+1}
$$

and, therefore,

$$
\int_{0}^{\bar{q}_{1}} \xi^{p} f_{1}(\xi) d \xi=O\left(\bar{q}^{p+1}\right)
$$

We also have, for all $i \geq 1$,

$$
f_{1}\left(\bar{q}_{i+1}\right) \int_{\bar{q}_{i}}^{\bar{q}_{i+1}}\left|\xi-\bar{r}_{i}\right|^{p} d \xi \leq \int_{\bar{q}_{i}}^{\bar{q}_{i+1}}\left|\xi-\bar{r}_{i}\right|^{p} f_{1}(\xi) d \xi \leq f_{1}\left(\bar{q}_{i}\right) \int_{\bar{q}_{i}}^{\bar{q}_{i+1}}\left|\xi-\bar{r}_{i}\right|^{p} d \xi
$$

with

$$
\int_{\bar{q}_{i}}^{\bar{q}_{i+1}}\left|\xi-\bar{r}_{i}\right|^{p} d \xi=\frac{\nu \bar{q}^{p+1}}{p+1} .
$$

In addition, we have the following inequalities:

$$
\begin{aligned}
& \forall \xi \in\left[\bar{q}_{i+1}, \bar{q}_{i+2}\right], \quad f_{1}(\xi) \leq f_{1}\left(\bar{q}_{i+1}\right) \\
\Rightarrow \quad & \int_{\bar{q}_{i+1}}^{\bar{q}_{i+2}} f_{1}(\xi) d \xi \leq \bar{q} f_{1}\left(\bar{q}_{i+1}\right)
\end{aligned}
$$


and, for all $i \geq 2$,

$$
\begin{gathered}
\forall \xi \in\left[\bar{q}_{i-1}, \bar{q}_{i}\right], \quad f_{1}\left(\bar{q}_{i}\right) \leq f_{1}(\xi) \\
\Rightarrow \quad \bar{q} f_{1}\left(\bar{q}_{i}\right) \leq \int_{\bar{q}_{i-1}}^{\bar{q}_{i}} f_{1}(\xi) d \xi .
\end{gathered}
$$

We deduce from (125), (126), (128) and (130) that

$$
\frac{\nu \bar{q}^{p}}{p+1} \int_{\bar{q}_{2}}^{\infty} f_{1}(\xi) d \xi \leq \sum_{i=1}^{\infty} \int_{\bar{q}_{i}}^{\bar{q}_{i+1}}\left|\xi-\bar{r}_{i}\right|^{p} f_{1}(\xi) d \xi \leq \frac{\nu \bar{q}^{p+1}}{p+1} f_{1}\left(\bar{q}_{1}\right)+\frac{\nu \bar{q}^{p}}{p+1} \int_{\bar{q}_{1}}^{\infty} f_{1}(\xi) d \xi
$$

which leads to

$$
\frac{\nu \bar{q}^{p}}{2(p+1)}\left(1-Q_{1 / \beta}\left(\bar{q}_{2}^{\beta}\right)\right) \leq \sum_{i=1}^{\infty} \int_{\bar{q}_{i}}^{\bar{q}_{i+1}}\left|\xi-\bar{r}_{i}\right|^{p} f_{1}(\xi) d \xi \leq \frac{\nu \bar{q}^{p+1}}{p+1} f_{1}(0)+\frac{\nu \bar{q}^{p}}{2(p+1)}\left(1-Q_{1 / \beta}\left(\bar{q}_{1}^{\beta}\right)\right) .
$$

We further know from (93) that, as $\bar{q} \rightarrow 0$, both $Q_{1 / \beta}\left(\bar{q}_{2}^{\beta}\right)$ and $Q_{1 / \beta}\left(\bar{q}_{1}^{\beta}\right)$ are $O(\bar{q})$. We conclude from (132) that

$$
\sum_{i=1}^{\infty} \int_{\bar{q}_{i}}^{\bar{q}_{i+1}}\left|\xi-\bar{r}_{i}\right|^{p} f_{1}(\xi) d \xi=\frac{\nu \bar{q}^{p}}{2(p+1)}(1+O(\bar{q}))
$$

where the local bound in $O(\bar{q})$ holds uniformly in $\nu$ (thus, in $\zeta$ ). Due to the fact that the integral in (124) does not depend on $\zeta$ and $\nu \geq 2^{-p}$, we have then, uniformly in $\zeta$,

$$
\int_{0}^{\bar{q}_{1}} \xi^{p} f_{1}(\xi) d \xi+\sum_{i=1}^{\infty} \int_{\bar{q}_{i}}^{\bar{q}_{i+1}}\left|\xi-\bar{r}_{i}\right|^{p} f_{1}(\xi) d \xi=\frac{\nu \bar{q}^{p}}{2(p+1)}(1+O(\bar{q}))
$$

which, combined with (122), yields the desired result.

\section{APPENDIX F}

\section{PROOF OF PROPOSITION 4}

According to Lemma 4 , there exists $\eta>0$ and $A>0$ such that, for all $\zeta \in[-1 / 2,1 / 2]$ and $\bar{q} \in(0, \eta)$,

$$
\bar{d}_{p, \zeta}(\bar{q})=\frac{\nu \bar{q}^{p}}{p+1}\left(1+\bar{q} a_{\zeta}(\bar{q})\right)
$$

and $\left|a_{\zeta}(\bar{q})\right| \leq A$. By noticing that, for all $\theta \geq-1,\left|(1+\theta)^{1 / p}-1\right| \leq|\theta|$, we deduce that

$$
\left|\frac{1}{\bar{q}}\left(\frac{(p+1) \bar{d}_{p, \zeta}(\bar{q})}{\nu}\right)^{1 / p}-1\right|=\left|\left(1+\bar{q} a_{\zeta}(\bar{q})\right)^{1 / p}-1\right| \leq \bar{q}\left|a_{\zeta}(\bar{q})\right| \leq A \bar{q} .
$$

This shows that

$$
\left(\bar{d}_{p, \zeta}(\bar{q})\right)^{1 / p}=\bar{q}\left(\frac{\nu}{p+1}\right)^{1 / p}\left(1+\bar{q} \widetilde{a}_{\zeta}(\bar{q})\right)
$$

where $\left|\widetilde{a}_{\zeta}(\bar{q})\right| \leq A$. Without loss of generality, one can choose $\eta<1 / A$ so that $1+\bar{q} \widetilde{a}_{\zeta}(\bar{q})>0$.

Let us first assume that $\bar{d}_{p, \zeta}(\bar{q}) \leq \bar{D} / \epsilon$ where $\bar{D}$ has been chosen small enough so that $\bar{q}<\eta$. It follows from (137) that we have the equivalence:

$$
\epsilon \bar{d}_{p, \zeta}(\bar{q}) \leq \bar{D} \quad \Leftrightarrow \quad \bar{q} \leq\left(\frac{(p+1) \bar{D}}{\nu \epsilon}\right)^{1 / p}\left(1+\bar{q} \widetilde{a}_{\zeta}(\bar{q})\right)^{-1} .
$$

Since $\nu \geq 2^{-p}$, this entails that

$$
\bar{q} \leq\left(\frac{(p+1) \bar{D}}{\nu \epsilon}\right)^{1 / p}(1-\eta A)^{-1} \leq 2\left(\frac{(p+1) \bar{D}}{\epsilon}\right)^{1 / p}(1-\eta A)^{-1} .
$$


Consequently, by defining

$$
\gamma_{1}(\bar{D})=2 A\left(\frac{(p+1) \bar{D}}{\epsilon}\right)^{1 / p}(1-\eta A)^{-1}
$$

we have

$$
\bar{q} \widetilde{a}_{\zeta}(\bar{q}) \geq-\gamma_{1}(\bar{D})
$$

So, by choosing $\bar{D}$ small enough, $1-\gamma_{1}(\bar{D})>0$ and (138) leads to

$$
\bar{q} \leq \frac{\rho_{1}(\bar{D})}{\nu^{1 / p}}
$$

where

$$
\rho_{1}(\bar{D})=\left(\frac{(p+1) \bar{D}}{\epsilon}\right)^{1 / p}\left(1-\gamma_{1}(\bar{D})\right)^{-1}
$$

In other words, we have shown that, provided that $\bar{D}$ is small enough,

$$
\left\{\bar{q} \mid \epsilon \bar{d}_{p, \zeta}(\bar{q}) \leq \bar{D}\right\} \subset\left\{\bar{q} \mid \bar{q} \leq \nu^{-1 / p} \rho_{1}(\bar{D})\right\}
$$

From (38), it can be concluded that

$$
R_{p, \zeta}(\epsilon, \bar{D}) \geq \inf _{\bar{q} \leq \nu^{-1 / p} \rho_{1}(\bar{D})} H_{g}(\epsilon, \bar{q})=H_{g}\left(\epsilon, \nu^{-1 / p} \rho_{1}(\bar{D})\right) .
$$

where Remark 2(i) has been used for the last equality. By noticing that

$$
\rho_{1}(\bar{D})=\left(\frac{(p+1) \bar{D}}{\epsilon}\right)^{1 / p}\left(1+O\left(\bar{D}^{1 / p}\right)\right)
$$

and using (29), we derive that

$$
R_{p, \zeta}(\epsilon, \bar{D}) \geq H_{\epsilon}+\epsilon\left(h_{\beta}(1)-\frac{1}{p} \ln \left(\frac{(p+1) \bar{D}}{\epsilon \nu}\right)+\mathbf{1}_{(0,1)}(\epsilon)\left(\frac{(p+1) \bar{D}}{\epsilon \nu}\right)^{1 / p} \frac{\beta \ln \bar{D}}{2 p \Gamma(1 / \beta)}\right)+O\left(\bar{D}^{1 / p}\right) .
$$

Let us now assume that $\bar{q}$ is such that

$$
\bar{q} \leq \frac{\rho_{2}(\bar{D})}{\nu^{1 / p}}
$$

where

$$
\rho_{2}(\bar{D})=\left(\frac{(p+1) \bar{D}}{\epsilon}\right)^{1 / p}\left(1+\gamma_{2}(\bar{D})\right)^{-1}
$$

and

$$
\gamma_{2}(\bar{D})=2 A\left(\frac{(p+1) \bar{D}}{\epsilon}\right)^{1 / p}
$$

We have then

$$
\begin{aligned}
& \gamma_{2}(\bar{D})\left(1+\gamma_{2}(\bar{D})\right) \geq 2 A\left(\frac{(p+1) \bar{D}}{\epsilon}\right)^{1 / p} \geq A\left(\frac{(p+1) \bar{D}}{\nu \epsilon}\right)^{1 / p} \\
\Leftrightarrow & \gamma_{2}(\bar{D}) \geq A\left(\frac{(p+1) \bar{D}}{\nu \epsilon}\right)^{1 / p}\left(1+\gamma_{2}(\bar{D})\right)^{-1} .
\end{aligned}
$$

It is clear from (148) and (149) that, by choosing $\bar{D}$ small enough, we have $\bar{q}<\eta$. We deduce from these two equations and (152) that

$$
\gamma_{2}(\bar{D}) \geq A \bar{q} \geq \widetilde{a}_{\zeta}(\bar{q}) \bar{q}
$$


which yields

$$
\bar{q} \leq\left(\frac{(p+1) \bar{D}}{\nu \epsilon}\right)^{1 / p}\left(1+\bar{q} \widetilde{a}_{\zeta}(\bar{q})\right)^{-1} .
$$

By using (138), we conclude that, for $\bar{D}$ small enough,

$$
\left\{\bar{q} \mid \bar{q} \leq \nu^{-1 / p} \rho_{2}(\bar{D})\right\} \subset\left\{\bar{q} \mid \epsilon \bar{d}_{p, \zeta}(\bar{q}) \leq \bar{D}\right\}
$$

Consequently, by using (38) and Remark 2(i),

$$
R_{p, \zeta}(\epsilon, \bar{D}) \leq \inf _{\bar{q} \leq \nu^{-1 / p} \rho_{2}(\bar{D})} H_{g}(\epsilon, \bar{q})=H_{g}\left(\epsilon, \nu^{-1 / p} \rho_{2}(\bar{D})\right) .
$$

Since

$$
\rho_{2}(\bar{D})=\left(\frac{(p+1) \bar{D}}{\epsilon}\right)^{1 / p}\left(1+O\left(\bar{D}^{1 / p}\right)\right)
$$

we deduce from (29) that

$$
R_{p, \zeta}(\epsilon, \bar{D}) \leq H_{\epsilon}+\epsilon\left(h_{\beta}(1)-\frac{1}{p} \ln \left(\frac{(p+1) \bar{D}}{\epsilon \nu}\right)+\mathbf{1}_{(0,1)}(\epsilon)\left(\frac{(p+1) \bar{D}}{\epsilon \nu}\right)^{1 / p} \frac{\beta \ln \bar{D}}{2 p \Gamma(1 / \beta)}\right)+O\left(\bar{D}^{1 / p}\right) .
$$

Combining (147) and (158) yields (42).

We proceed similarly to prove (43). Instead of (145), we have just to use the fact that, for $\bar{D}$ small enough,

$$
R_{p}(\epsilon, \bar{D}) \geq \inf _{\substack{-1 / 2 \leq \zeta \leq 0 \\ \bar{q} \leq \nu^{-1 / p} \rho_{1}(\bar{D})}} H_{g}(\epsilon, \bar{q})=H_{g}\left(\epsilon, 2 \rho_{1}(\bar{D})\right)
$$

since the minimum value of $\nu$ is reached when $\zeta=0$ and it is equal to $2^{-p}$. In the same way, (156) has to be replaced by

$$
R_{p}(\epsilon, \bar{D}) \leq \inf _{\substack{-1 / 2 \leq \zeta \leq 0 \\ \bar{q} \leq \nu^{-1 / p} \rho_{2}(\bar{D})}} H_{g}(\epsilon, \bar{q})=H_{g}\left(\epsilon, 2 \rho_{2}(\bar{D})\right)
$$

\section{APPENDIX G}

\section{TECHNICAL RESULTS FOR THE PROOF OF PROPOSITION 5}

In this appendix, we provide some preliminary results for the proof of Proposition 5.

Lemma 5: For all $n \geq 1$, we have, as $\bar{q} \rightarrow \infty$,

$$
\omega^{p / \beta} \sum_{i=n}^{\infty} \int_{\left(i-\frac{1}{2}\right) q}^{\left(i+\frac{1}{2}\right) q}\left|\xi-r_{i}\right|^{p} f(\xi) d \xi \leq \frac{(2 n-1) \bar{q}^{p+1} e^{-\bar{q}_{n}^{\beta}}}{4 \Gamma(1 / \beta) \tilde{q}_{n}^{\beta}}\left(1+O\left(\frac{1}{\bar{q}^{2 \beta}}\right)\right)
$$

where $\tilde{q}_{n}=\left(\bar{q}_{n}^{\beta}+1-1 / \beta\right)^{1 / \beta}$.

Proof: For all $i \geq n$, we have the inequality:

$$
\omega^{p / \beta} \int_{\left(i-\frac{1}{2}\right) q}^{\left(i+\frac{1}{2}\right) q}\left|\xi-r_{i}\right|^{p} f(\xi) d \xi \leq \bar{q}^{p} \mathbf{p}_{i}
$$

and, consequently,

$$
\omega^{p / \beta} \sum_{i=n}^{\infty} \int_{\left(i-\frac{1}{2}\right) q}^{\left(i+\frac{1}{2}\right) q}\left|\xi-r_{i}\right|^{p} f(\xi) d \xi \leq \bar{q}^{p}\left(\frac{1}{2}-\mathrm{P}(0 \leq \tilde{X} \leq(n-1 / 2) q)\right)=\frac{\bar{q}^{p}}{2}\left(1-Q_{1 / \beta}\left(\bar{q}_{n}^{\beta}\right)\right)
$$

where $\tilde{X}$ is a GG random variable distributed according to (1). The result then follows from (94). 
Lemma 6: As $\bar{q} \rightarrow \infty$, we have

$$
\bar{d}_{p, \zeta}(\bar{q})=\frac{1}{\Gamma(1 / \beta)}\left(\Gamma\left(\frac{p+1}{\beta}\right) Q_{(p+1) / \beta}\left(\bar{q}_{1}^{\beta}\right)+\beta \int_{\bar{q}_{1}}^{\infty}\left|\xi-\bar{r}_{1}\right|^{p} e^{-\xi^{\beta}} d \xi\right)+O\left(\bar{q}^{p+1-\beta} e^{-\bar{q}_{2}^{\beta}}\right)
$$

Proof: According to (36), the first integral term in the expression of $\bar{d}_{p, \zeta}(\bar{q}) / 2$ is

$$
\omega^{p / \beta} \int_{0}^{\frac{q}{2}} \xi^{p} f(\xi) d \xi=\frac{\Gamma((p+1) / \beta)}{2 \Gamma(1 / \beta)} Q_{(p+1) / \beta}\left(\bar{q}_{1}^{\beta}\right)
$$

The second term in the expression of $\bar{d}_{p, \zeta}(\bar{q}) / 2$ is

$$
\omega^{p / \beta} \int_{\frac{q}{2}}^{\frac{3 q}{2}}\left|\xi-r_{1}\right|^{p} f(\xi) d \xi=\frac{\beta}{2 \Gamma(1 / \beta)}\left(\int_{\bar{q}_{1}}^{\infty}\left|\xi-\bar{r}_{1}\right|^{p} e^{-\xi^{\beta}} d \xi-\int_{\bar{q}_{2}}^{\infty}\left(\xi-\bar{r}_{1}\right)^{p} e^{-\xi^{\beta}} d \xi\right) .
$$

In addition, by making the change of variable $\theta=\xi^{\beta}-\bar{q}_{2}^{\beta}$, we find that

$$
\beta \bar{q}_{2}^{-p-1+\beta} e^{\bar{q}_{2}^{\beta}} \int_{\bar{q}_{2}}^{\infty}\left(\xi-\bar{r}_{1}\right)^{p} e^{-\xi^{\beta}} d \xi=\int_{0}^{\infty}\left(\left(\frac{\theta}{\bar{q}_{2}^{\beta}}+1\right)^{1 / \beta}-\frac{2}{3}(1+\zeta)\right)^{p}\left(\frac{\theta}{\bar{q}_{2}^{\beta}}+1\right)^{1 / \beta-1} e^{-\theta} d \theta
$$

Assuming that $\bar{q} \geq 2 / 3$, we have:

$$
\forall \theta \geq 0, \quad 1 \leq\left(\frac{\theta}{\bar{q}_{2}^{\beta}}+1\right)^{1 / \beta} \leq(\theta+1)^{1 / \beta}
$$

and, since $p \geq 1$ and $\beta \geq 1$,

$$
0 \leq \int_{0}^{\infty}\left(\left(\frac{\theta}{\bar{q}_{2}^{\beta}}+1\right)^{1 / \beta}-\frac{2}{3}(1+\zeta)\right)^{p}\left(\frac{\theta}{\bar{q}_{2}^{\beta}}+1\right)^{1 / \beta-1} e^{-\theta} d \theta \leq \int_{0}^{\infty}\left((\theta+1)^{1 / \beta}-\frac{2}{3}(1+\zeta)\right)^{p} e^{-\theta} d \theta<\infty
$$

This shows that

$$
\int_{\bar{q}_{2}}^{\infty}\left(\xi-\bar{r}_{1}\right)^{p} e^{-\xi^{\beta}} d \xi=O\left(\bar{q}^{p+1-\beta} e^{-\bar{q}_{2}^{\beta}}\right)
$$

By invoking now Lemma 5 with $n=2$, it can be claimed that

$$
\omega^{p / \beta} \sum_{i=2}^{\infty} \int_{\left(i-\frac{1}{2}\right) q}^{\left(i+\frac{1}{2}\right) q}\left|\xi-r_{i}\right|^{p} f(\xi) d \xi=O\left(\bar{q}^{p+1-\beta} e^{-\bar{q}_{2}^{\beta}}\right) .
$$

By using (36) in conjunction with (165), (166), (170) and (171), the expression in (164) is obtained.

Lemma 7: Let $\lambda \geq 1$ and let

$$
\forall \alpha \in \mathbb{R}_{+}, \quad I(\alpha)=\int_{0}^{\infty}\left|(\alpha \theta+1)^{1 / \beta}-\lambda\right|^{p}(\alpha \theta+1)^{1 / \beta-1} e^{-\theta} d \theta .
$$

Then, as $\alpha \rightarrow 0$

$$
I(\alpha)= \begin{cases}(\lambda-1)^{p}-\frac{\alpha}{\beta}(\lambda-1)^{p-1}(p+(\beta-1)(\lambda-1))+O\left(\alpha^{2}\right) & \text { if } \lambda \neq 1 \\ \frac{\alpha}{\beta}+O\left(\alpha^{2}\right) & \text { if } \lambda=1 \text { and } p=1 \\ O\left(\alpha^{p}\right) & \text { if } \lambda=1 \text { and } p>1 .\end{cases}
$$

Proof: We distinguish the two cases : $\lambda>1$ and $\lambda=1$. 
(i) Case $\lambda>1$ : Let us assume that $0<\alpha<1$. We can decompose the integral of interest as follows:

$$
I(\alpha)=\int_{0}^{\frac{\lambda^{\beta}-1}{\alpha}}\left(\lambda-(\alpha \theta+1)^{1 / \beta}\right)^{p}(\alpha \theta+1)^{1 / \beta-1} e^{-\theta} d \theta+\int_{\frac{\lambda^{\beta}-1}{\alpha}}^{\infty}\left((\alpha \theta+1)^{1 / \beta}-\lambda\right)^{p}(\alpha \theta+1)^{1 / \beta-1} e^{-\theta} d \theta .
$$

Let us now focus on the first integral on the right-hand side of (174). This reads

$$
I_{1}(\alpha)=\int_{0}^{\frac{\lambda^{\beta}-1}{\alpha}} \varphi(\alpha \theta) e^{-\theta} d \theta
$$

where

$$
\varphi: \mathbb{R}_{+} \rightarrow \mathbb{R}: u \mapsto\left|\lambda-(u+1)^{1 / \beta}\right|^{p}(u+1)^{1 / \beta-1}
$$

The latter function is continuously differentiable ${ }^{8}$ on $\left[0, \lambda^{\beta}-1\right]$ and it is twice differentiable on $\left[0, \lambda^{\beta}-1\right)$. Its first and second derivatives on the considered intervals are given by

$$
\varphi^{\prime}(u)=-\frac{1}{\beta} v(u)^{1-2 \beta}(\lambda-v(u))^{p-1}((p+1-\beta) v(u)+(\beta-1) \lambda)
$$

and

$\varphi^{\prime \prime}(u)=\frac{1}{\beta^{2}} v(u)^{1-3 \beta}(\lambda-v(u))^{p-2}\left((p+1-\beta)(p+1-2 \beta)(v(u))^{2}+(\beta-1)(3 p+2-4 \beta) \lambda v(u)+(\beta-1)(2 \beta-1) \lambda^{2}\right)$

where $v(u)=(u+1)^{1 / \beta}$. By performing a Taylor-Mc Laurin expansion of $\varphi$, we get, for all $\theta \in\left[0,\left(\lambda^{\beta}-1\right) / \alpha\right]$,

$$
\begin{aligned}
\varphi(\alpha \theta) & =\varphi(0)+\varphi^{\prime}(0) \alpha \theta+\frac{1}{2} \varphi^{\prime \prime}(\mu)(\alpha \theta)^{2} \\
& =(\lambda-1)^{p}-\frac{1}{\beta}(\lambda-1)^{p-1}(p+(\beta-1)(\lambda-1)) \alpha \theta+\frac{1}{2} \varphi^{\prime \prime}(\mu) \alpha^{2} \theta^{2}
\end{aligned}
$$

where $0<\mu<\alpha \theta$. In addition, since it has been assumed that $\alpha<1$, we have $1<v(\mu)<(\alpha \theta+1)^{1 / \beta}<$ $(\theta+1)^{1 / \beta}$ (which implies $0<v(\mu)^{1-3 \beta}<1$ ) and

$$
\left|\varphi^{\prime \prime}(\mu)\right| \leq \rho_{\alpha}(\theta)
$$

where the expression of the upper bound $\rho_{\alpha}(\theta)$ depends on the value of the real parameter $p$ :

(a) When $p \geq 2,(\lambda-v(\mu))^{p-2} \leq(\lambda-1)^{p-2}$ and one can take

$$
\rho_{\alpha}(\theta)=\frac{1}{\beta^{2}}(\lambda-1)^{p-2}\left((p+1-\beta)|p+1-2 \beta|(\theta+1)^{2 / \beta}+(\beta-1)(3 p+2-4 \beta) \lambda(\theta+1)^{1 / \beta}+(\beta-1)(2 \beta-1) \lambda^{2}\right) .
$$

(b) When $p=1$, the second derivative takes the following simplified form:

$$
\varphi^{\prime \prime}(\mu)=\frac{\beta-1}{\beta^{2}} v(\mu)^{1-3 \beta}(2(2-\beta) v(\mu)+(2 \beta-1) \lambda)
$$

thus yielding

$$
\rho_{\alpha}(\theta)=\frac{\beta-1}{\beta^{2}}\left(2(2-\beta)(\theta+1)^{1 / \beta}+(2 \beta-1) \lambda\right)
$$

${ }^{8} \mathrm{We}$ consider one-sided derivatives at the interval boundaries. 
(c) When $1<p<2$, we take

$$
\begin{aligned}
\rho_{\alpha}(\theta)=\frac{1}{\beta^{2}}\left(\lambda-(\alpha \theta+1)^{1 / \beta}\right)^{p-2} & \left((p+1-\beta)|p+1-2 \beta|(\alpha \theta+1)^{2 / \beta}\right. \\
& \left.+(\beta-1)|3 p+2-4 \beta| \lambda(\alpha \theta+1)^{1 / \beta}+(\beta-1)(2 \beta-1) \lambda^{2}\right) .
\end{aligned}
$$

Now, it can be deduced from (175), (179) and (180) that

$$
\left|I_{1}(\alpha)-(\lambda-1)^{p} \int_{0}^{\frac{\lambda^{\beta}-1}{\alpha}} e^{-\theta} d \theta+\frac{\alpha}{\beta}(\lambda-1)^{p-1}(p+(\beta-1)(\lambda-1)) \int_{0}^{\frac{\lambda^{\beta}-1}{\alpha}} \theta e^{-\theta} d \theta\right| \leq \frac{\alpha^{2}}{2} \int_{0}^{\frac{\lambda^{\beta}-1}{\alpha}} \theta^{2} \rho_{\alpha}(\theta) e^{-\theta} d \theta .
$$

This leads to

$$
\left|I_{1}(\alpha)-(\lambda-1)^{p}+\frac{\alpha}{\beta}(\lambda-1)^{p-1}(p+(\beta-1)(\lambda-1))+O\left(\frac{e^{-\left(\lambda^{\beta}-1\right) / \alpha}}{\alpha}\right)\right| \leq \frac{\alpha^{2}}{2} \int_{0}^{\infty} \theta^{2} \rho_{\alpha}(\theta) e^{-\theta} d \theta .
$$

In the cases when $p=1$ or $p \geq 2$, it can be verified from the expressions (181) and (182) that the integral in the upper bound is not dependent on $\alpha$ and it is convergent. This implies:

$$
I_{1}(\alpha)=(\lambda-1)^{p}-\frac{\alpha}{\beta}(\lambda-1)^{p-1}(p+(\beta-1)(\lambda-1))+O\left(\alpha^{2}\right) .
$$

In the case when $1<p<2$, we decompose the upper bound integral in (185) as

$$
\int_{0}^{\frac{\lambda^{\beta}-1}{\alpha}} \theta^{2} \rho_{\alpha}(\theta) e^{-\theta} d \theta=\int_{0}^{\frac{\eta}{\alpha}} \theta^{2} \rho_{\alpha}(\theta) e^{-\theta} d \theta+\int_{\frac{\eta}{\alpha}}^{\frac{\lambda^{\beta}-1}{\alpha}} \theta^{2} \rho_{\alpha}(\theta) e^{-\theta} d \theta
$$

where $0<\eta<\lambda^{\beta}-1$. From (184), we find that, for all $\theta \in[0, \eta / \alpha]$,

$$
\begin{aligned}
\rho_{\alpha}(\theta) \leq \bar{\rho}=\frac{1}{\beta^{2}}\left(\lambda-(\eta+1)^{1 / \beta}\right)^{p-2}( & (p+1-\beta)|p+1-2 \beta|(\eta+1)^{2 / \beta} \\
& \left.+(\beta-1)|3 p+2-4 \beta| \lambda(\eta+1)^{1 / \beta}+(\beta-1)(2 \beta-1) \lambda^{2}\right)
\end{aligned}
$$

and, consequently,

$$
\int_{0}^{\frac{\eta}{\alpha}} \theta^{2} \rho_{\alpha}(\theta) e^{-\theta} d \theta \leq \bar{\rho} \int_{0}^{\infty} \theta^{2} e^{-\theta} d \theta=2 \bar{\rho} .
$$

Besides, after the change of variable $\tau=\alpha \theta$, the second integral in the right-hand side of (188) can be rewritten as

$$
\int_{\frac{\eta}{\alpha}}^{\frac{\lambda^{\beta}-1}{\alpha}} \theta^{2} \rho_{\alpha}(\theta) e^{-\theta} d \theta=\int_{\eta}^{\lambda^{\beta}-1} \tau^{2} \rho_{1}(\tau) \frac{e^{-\tau / \alpha}}{\alpha^{3}} d \tau \leq \frac{e^{-\eta / \alpha}}{\alpha^{3}} \int_{\eta}^{\lambda^{\beta}-1} \tau^{2} \rho_{1}(\tau) d \tau .
$$

The latter integral is convergent since $\left(\lambda-(\tau+1)^{1 / \beta}\right)^{p-2}=O\left(\left(\lambda^{\beta}-1-\tau\right)^{p-2}\right)$, as $\tau \rightarrow \lambda^{\beta}-1$, and $-1<p-2<0$. By using this fact in combination with (190) and (188), we conclude that, as $\alpha \rightarrow 0$,

$$
\int_{0}^{\frac{\lambda^{\beta}-1}{\alpha}} \theta^{2} \rho_{\alpha}(\theta) e^{-\theta} d \theta=O(1)
$$

which, together with (185), allows us to claim that (187) still holds when $1<p<2$. 
We finally observe that the last term in (174) can be re-expressed as

$$
I_{2}(\alpha)=e^{-\frac{\lambda^{\beta}-1}{\alpha}} \int_{0}^{\infty} \varphi\left(\alpha \theta+\lambda^{\beta}-1\right) e^{-\theta} d \theta
$$

where, for $\alpha<1$,

$$
0 \leq \varphi\left(\alpha \theta+\lambda^{\beta}-1\right) \leq\left(\left(\theta+\lambda^{\beta}\right)^{1 / \beta}-\lambda\right)^{p}
$$

since $\lambda \geq 1 \Rightarrow\left(\alpha \theta+\lambda^{\beta}\right)^{1 / \beta-1} \leq 1$. This yields

$$
I_{2}(\alpha)=O\left(e^{-\frac{\lambda^{\beta}-1}{\alpha}}\right) .
$$

As $\lim _{\alpha \rightarrow 0} \alpha^{-2} e^{-\frac{\lambda^{\beta}-1}{\alpha}}=0$, we deduce from (174), (187) and (195) that (173) holds.

(ii) Case $\lambda=1$ : We have then, for all $\alpha>0$,

$$
I(\alpha)=\int_{0}^{\infty}\left((\alpha \theta+1)^{1 / \beta}-1\right)^{p}(\alpha \theta+1)^{1 / \beta-1} e^{-\theta} d \theta .
$$

(a) When $p=1$, this integral can be expressed as

$$
\begin{aligned}
I(\alpha) & =\alpha^{1 / \beta-1} e^{1 / \alpha} \int_{1 / \alpha}^{\infty}\left(\alpha^{1 / \beta} u^{1 / \beta}-1\right)^{p} u^{1 / \beta-1} e^{-u} d u \\
& =e^{1 / \alpha}\left(\alpha^{2 / \beta-1} \int_{1 / \alpha}^{\infty} u^{2 / \beta-1} e^{-u} d u-\alpha^{1 / \beta-1} \int_{1 / \alpha}^{\infty} u^{1 / \beta-1} e^{-u} d u\right)
\end{aligned}
$$

where the change of variable $u=\theta+1 / \alpha$ has been performed to get the first equality. From (8) and (94), we deduce that

$$
I(\alpha)=\frac{\alpha^{-1}}{\alpha^{-1}-\frac{2}{\beta}+1}-\frac{\alpha^{-1}}{\alpha^{-1}-\frac{1}{\beta}+1}+O\left(\alpha^{2}\right)=\frac{\alpha}{\beta}+O\left(\alpha^{2}\right) .
$$

(b) Let us now consider the subcase $p>1$. By integration by parts, (196) becomes

$$
I(\alpha)=\frac{\beta}{(p+1) \alpha} \int_{0}^{\infty}\left((\alpha \theta+1)^{1 / \beta}-1\right)^{p+1} e^{-\theta} d \theta .
$$

Since $\beta \geq 1$, for every $\theta \geq 0,(\alpha \theta+1)^{1 / \beta} \leq \alpha \theta+1$, which allows us to upper bound $I(\alpha) \geq 0$ by

$$
\bar{I}(\alpha)=\frac{\beta \alpha^{p}}{(p+1)} \int_{0}^{\infty} \theta^{p+1} e^{-\theta} d \theta=\beta \Gamma(p+1) \alpha^{p} .
$$

Consequently, $I(\alpha)=O\left(\alpha^{p}\right)$.

In summary, we have proven that (173) also holds when $\lambda=1$.

\section{APPENDIX H}

\section{PROOF OF PROPOSITION 6}

We proceed similarly to the proof of [17].

Proof of (63) and (64): Let us first calculate

$$
\limsup _{\bar{D} \rightarrow\left(\epsilon \mu_{p}\right)^{-}} \frac{R_{p, \zeta}(\epsilon, \bar{D})}{\epsilon \mu_{p}-\bar{D}}=\alpha \in(-\infty, \infty] .
$$


The above equality means that

$$
\lim _{\eta \rightarrow 0^{+}} \sup \left\{\frac{R_{p, \zeta}(\epsilon, \bar{D})}{\epsilon \mu_{p}-\bar{D}}, \epsilon\left(\mu_{p}-\eta\right)<\bar{D}<\epsilon \mu_{p}\right\}=\alpha .
$$

In addition, $\bar{d}_{p, \zeta}$ is a continuous function and, according to (48),

$$
\lim _{\bar{q} \rightarrow \infty} \bar{d}_{p, \zeta}(\bar{q})=\mu_{p}
$$

and (see Remark 4(iii))

$$
\exists \tau>0 \quad \text { such that } \forall \bar{q}>\tau, \bar{d}_{p, \zeta}(\bar{q})<\mu_{p} .
$$

It can then be deduced from (202) that

$$
\limsup _{\bar{q} \rightarrow \infty} \frac{R_{p, \zeta}\left(\epsilon, \epsilon \bar{d}_{p, \zeta}(\bar{q})\right)}{\mu_{p}-\bar{d}_{p, \zeta}(\bar{q})}=\epsilon \alpha
$$

Using now (38), we have

$$
\limsup _{\bar{q} \rightarrow \infty} \frac{R_{p, \zeta}\left(\epsilon, \epsilon \bar{d}_{p, \zeta}(\bar{q})\right)}{\mu_{p}-\bar{d}_{p, \zeta}(\bar{q})} \leq \limsup _{\bar{q} \rightarrow \infty} \frac{H_{g}(\epsilon, \bar{q})}{\mu_{p}-\bar{d}_{p, \zeta}(\bar{q})}=\lim _{\bar{q} \rightarrow \infty} \frac{H_{g}(\epsilon, \bar{q})}{\mu_{p}-\bar{d}_{p, \zeta}(\bar{q})}
$$

where the last limit can be derived from (61) and (62). In summary, we have proved that

$$
\epsilon \limsup _{\bar{D} \rightarrow\left(\epsilon \mu_{p}\right)^{-}} \frac{R_{p, \zeta}(\epsilon, \bar{D})}{\epsilon \mu_{p}-\bar{D}} \leq \lim _{\bar{q} \rightarrow \infty} \frac{H_{g}(\epsilon, \bar{q})}{\mu_{p}-\bar{d}_{p, \zeta}(\bar{q})} .
$$

Let us now set $\bar{D}<\epsilon \mu_{p}$. From the definition of $R_{p, \zeta}$ in (38), for all $\eta>0$ there exists $\bar{q}_{\eta}$ such that $\epsilon \bar{d}_{p, \zeta}\left(\bar{q}_{\eta}\right) \leq \bar{D}$ and $H_{g}\left(\epsilon, \bar{q}_{\eta}\right)-\eta<R_{p, \zeta}(\epsilon, \bar{D}) \leq H_{g}\left(\epsilon, \bar{q}_{\eta}\right)$. This holds in particular when $\eta=\eta(\bar{D})=\left(\epsilon \mu_{p}-\bar{D}\right)^{2}$. Consequently,

$$
\frac{R_{p, \zeta}(\epsilon, \bar{D})}{\epsilon \mu_{p}-\bar{D}}>\frac{H_{g}\left(\epsilon, \bar{q}_{\eta(\bar{D})}\right)-\eta(\bar{D})}{\epsilon \mu_{p}-\bar{D}}
$$

and

$$
\liminf _{\bar{D} \rightarrow\left(\epsilon \mu_{p}\right)^{-}} \frac{R_{p, \zeta}(\epsilon, \bar{D})}{\epsilon \mu_{p}-\bar{D}} \geq \liminf _{\bar{D} \rightarrow\left(\epsilon \mu_{p}\right)^{-}} \frac{H_{g}\left(\epsilon, \bar{q}_{\eta(\bar{D})}\right)-\eta(\bar{D})}{\epsilon \mu_{p}-\bar{D}}=\liminf _{\bar{D} \rightarrow\left(\epsilon \mu_{p}\right)^{-}} \frac{H_{g}\left(\epsilon, \bar{q}_{\eta(\bar{D})}\right)}{\epsilon \mu_{p}-\bar{D}} .
$$

Furthermore, we have

$$
0<\frac{1}{\mu_{p}-\bar{d}_{p, \zeta}\left(\bar{q}_{\eta(\bar{D})}\right)} \leq \frac{\epsilon}{\epsilon \mu_{p}-\bar{D}}
$$

which, combined with (209), yields

$$
\epsilon \liminf _{\bar{D} \rightarrow\left(\epsilon \mu_{p}\right)^{-}} \frac{R_{p, \zeta}(\epsilon, \bar{D})}{\epsilon \mu_{p}-\bar{D}} \geq \liminf _{\bar{D} \rightarrow\left(\epsilon \mu_{p}\right)^{-}} \frac{H_{g}\left(\epsilon, \bar{q}_{\eta(\bar{D})}\right)}{\mu_{p}-\bar{d}_{p, \zeta}\left(\bar{q}_{\eta(\bar{D})}\right)} .
$$

It can then be noticed that $\lim _{\bar{D} \rightarrow\left(\epsilon \mu_{p}\right)^{-}} R_{p, \zeta}(\epsilon, \bar{D})=0$ (from (38), (204) and the fact that $\left.\lim _{\bar{q} \rightarrow \infty} H_{g}(\epsilon, \bar{q})=0\right)$ ), which implies that $\lim _{\bar{D} \rightarrow\left(\epsilon \mu_{p}\right)^{-}} H_{g}\left(\epsilon, \bar{q}_{\eta(\bar{D})}\right)=0$ (since $0<H_{g}\left(\epsilon, \bar{q}_{\eta(\bar{D})}\right) \leq R_{p, \zeta}(\epsilon, \bar{D})+\eta(\bar{D})$ ). Thus, by invoking the monotonicity of $H_{g}(\epsilon, \cdot)$, we get $\lim _{\bar{D} \rightarrow\left(\epsilon \mu_{p}\right)-} \bar{q}_{\eta(\bar{D})}=\infty$. This allows us to deduce from (211) that

$$
\epsilon \liminf _{\bar{D} \rightarrow\left(\epsilon \mu_{p}\right)^{-}} \frac{R_{p, \zeta}(\epsilon, \bar{D})}{\epsilon \mu_{p}-\bar{D}} \geq \lim _{\bar{q} \rightarrow \infty} \frac{H_{g}(\epsilon, \bar{q})}{\mu_{p}-\bar{d}_{p, \zeta}(\bar{q})}
$$

By combining (207) and (212), we conclude that

$$
\epsilon \lim _{\bar{D} \rightarrow\left(\epsilon \mu_{p}\right)^{-}} \frac{R_{p, \zeta}(\epsilon, \bar{D})}{\epsilon \mu_{p}-\bar{D}}=\lim _{\bar{q} \rightarrow \infty} \frac{H_{g}(\epsilon, \bar{q})}{\mu_{p}-\bar{d}_{p, \zeta}(\bar{q})} .
$$


Eqs. (63) and (64) then straighforwardly follow from (61) and (62).

Proof of (65): Let us first consider the case $p<\beta$. Using Lemma 3 and proceeding similarly to the derivation of (211), we can claim that, for all $\bar{D} \in\left(0, \epsilon \mu_{p}\right)$, there exists $\bar{q}_{\eta(\bar{D})}^{\prime}>0$ and $\zeta_{\eta(\bar{D})} \in[-1 / 2,0]$ such that $\epsilon \bar{d}_{\left.p, \zeta_{\eta(\bar{D})}\right)}\left(\bar{q}_{\eta(\bar{D})}^{\prime}\right) \leq \bar{D}$ and

$$
\epsilon \liminf _{\bar{D} \rightarrow\left(\epsilon \mu_{p}\right)^{-}} \frac{R_{p}(\epsilon, \bar{D})}{\epsilon \mu_{p}-\bar{D}} \geq \liminf _{\bar{D} \rightarrow\left(\epsilon \mu_{p}\right)^{-}} \frac{H_{g}\left(\epsilon, \bar{q}_{\eta(\bar{D})}^{\prime}\right)}{\mu_{p}-\bar{d}_{p, \zeta_{\eta(\bar{D})}}\left(\bar{q}_{\eta(\bar{D})}^{\prime}\right)} .
$$

By using now Remark 4(iv), we obtain

$$
\epsilon \liminf _{\bar{D} \rightarrow\left(\epsilon \mu_{p}\right)^{-}} \frac{R_{p}(\epsilon, \bar{D})}{\epsilon \mu_{p}-\bar{D}} \geq \liminf _{\bar{D} \rightarrow\left(\epsilon \mu_{p}\right)^{-}} \frac{H_{g}\left(\epsilon, \bar{q}_{\eta(\bar{D})}^{\prime}\right)}{\mu_{p}-\underline{d}_{p}\left(\bar{q}_{\eta(\bar{D})}^{\prime}\right)} .
$$

In turn, the asymptotic forms of $H_{g}(\epsilon, \cdot)$ and $\underline{d}_{p}$ in (32) and (60) lead to

$$
\lim _{\bar{q} \rightarrow \infty} \frac{H_{g}(\epsilon, \bar{q})}{\mu_{p}-\underline{d}_{p}(\bar{q})}=\infty .
$$

Since $\bar{q}_{\eta(\bar{D})}^{\prime} \rightarrow \infty$ as $\bar{D} \rightarrow\left(\epsilon \mu_{p}\right)^{-}$, we deduce that

$$
\lim _{\bar{D} \rightarrow\left(\epsilon \mu_{p}\right)^{-}} \frac{R_{p}(\epsilon, \bar{D})}{\epsilon \mu_{p}-\bar{D}}=\infty .
$$

Let us now turn our attention to the case $p \geq \beta$. We have

$$
0 \leq \limsup _{\bar{D} \rightarrow\left(\epsilon \mu_{p}\right)^{-}} \frac{R_{p}(\epsilon, \bar{D})}{\epsilon \mu_{p}-\bar{D}}=\limsup _{\bar{D} \rightarrow\left(\epsilon \mu_{p}\right)^{-}} \frac{\inf _{-1 / 2 \leq \zeta \leq 0} R_{p, \zeta}(\epsilon, \bar{D})}{\epsilon \mu_{p}-\bar{D}} \leq \inf _{-1 / 2 \leq \zeta \leq 0} \limsup _{\bar{D} \rightarrow\left(\epsilon \mu_{p}\right)^{-}} \frac{R_{p, \zeta}(\epsilon, \bar{D})}{\epsilon \mu_{p}-\bar{D}} .
$$

By using (63) and (64), we find that:

(i) If $p>\beta$, then

$$
\lim _{\bar{D} \rightarrow\left(\epsilon \mu_{p}\right)^{-}} \frac{R_{p}(\epsilon, \bar{D})}{\epsilon \mu_{p}-\bar{D}}=0 .
$$

(ii) If $p=\beta$, then the infimum of the last term in (218) is attained when $\zeta=-1 / 2$ and we get

$$
\limsup _{\bar{D} \rightarrow\left(\epsilon \mu_{p}\right)^{-}} \frac{R_{p}(\epsilon, \bar{D})}{\epsilon \mu_{p}-\bar{D}} \leq 1 \text {. }
$$

Furthermore, for $\bar{D} \in\left(0, \epsilon \mu_{p}\right)$, we obviously have

$$
\frac{R_{p}(\epsilon, \bar{D})}{\epsilon \mu_{p}-\bar{D}} \geq \frac{\mathcal{R}_{p}(\epsilon, \bar{D})}{\epsilon \mu_{p}-\bar{D}}
$$

where $\mathcal{R}_{p}$ is the Shannon rate-distortion function defined by (45). By the chain rule [27], the mutual information in this expression is equal to

$$
\mathcal{I}(X ; \hat{X})=\mathcal{I}(Q ; \hat{X})+\epsilon \mathcal{I}\left(X^{(1)} ; \hat{X}^{(1)}\right)
$$

where

$$
Q= \begin{cases}0 & \text { if } X=0 \\ 1 & \text { otherwise }\end{cases}
$$


and $X^{(1)}$ (resp. $\hat{X}^{(1)}$ ) is a random variable distributed according to the distribution of $X$ (resp. $\hat{X}$ ) conditionally to $Q=1$. Since the mutual entropy is nonnegative, we derive the following lower bound for $\mathcal{R}_{p}$ (see [24] for more details):

$$
\mathcal{R}_{p}(\epsilon, \bar{D}) \geq \epsilon \inf _{\left\{\hat{X}^{(1)} \mid \mathrm{E}\left[\mid X^{(1)}-\hat{X}^{(1) \mid p]} \leq \omega-p / \beta \bar{D} / \epsilon\right\}\right.} \mathcal{I}\left(X^{(1)} ; \hat{X}^{(1)}\right)=\epsilon \mathcal{R}_{p}^{(1)}\left(\frac{\bar{D}}{\epsilon}\right)
$$

where $\mathcal{R}_{p}^{(1)}$ is the Shannon rate-distortion function of a GG random variable. The Shannon lower bound [31] for this latter rate-distortion function is given by:

$$
\mathcal{R}_{p}^{(1)}\left(\frac{\bar{D}}{\epsilon}\right) \geq h_{\beta}(1)-h_{p}(1)-\frac{1}{p} \ln \left(\frac{p \bar{D}}{\epsilon}\right) .
$$

When $\beta=p$, the above two inequalities reduce to

$$
\mathcal{R}_{p}(\epsilon, \bar{D}) \geq \frac{\epsilon}{p} \ln \left(\frac{\epsilon}{p \bar{D}}\right) .
$$

Then, by noticing that $\mu_{p}=\frac{\Gamma(1+1 / p)}{\Gamma(1 / p)}=1 / p$, we deduce from (221) and (226) that, as $\bar{D} \rightarrow\left(\epsilon \mu_{p}\right)^{-}$,

$$
1+o(1) \leq \frac{\mathcal{R}_{p}(\epsilon, \bar{D})}{\epsilon \mu_{p}-\bar{D}} \leq \frac{R_{p}(\epsilon, \bar{D})}{\epsilon \mu_{p}-\bar{D}}
$$

By combining this result with (220), we conclude that, when $p=\beta$,

$$
\lim _{\bar{D} \rightarrow\left(\epsilon \mu_{p}\right)^{-}} \frac{R_{p}(\epsilon, \bar{D})}{\epsilon \mu_{p}-\bar{D}}=\lim _{\bar{D} \rightarrow\left(\epsilon \mu_{p}\right)^{-}} \frac{\mathcal{R}_{p}(\epsilon, \bar{D})}{\epsilon \mu_{p}-\bar{D}}=1 .
$$

\section{REFERENCES}

[1] S. Mallat, "A theory for multiresolution signal decomposition: The wavelet representation," IEEE Trans. Pattern Anal. Machine Intell., vol. 11, pp. 674-693, 1989.

[2] P. Moulin and J. Liu, "Analysis of multiresolution image denoising schemes using generalized Gaussian and complexity priors," IEEE Trans. on Information Theory, vol. 45, no. 3, pp. 909-919, 1999.

[3] A. Antoniadis, D. Leporini, and J.-C. Pesquet, "Wavelet thresholding for some classes of non-Gaussian noise," Statistica Neerlandica, vol. 56, no. 4, pp. 434-453, 2002.

[4] W. Szepanski, " $\Delta$-entropy and rate distortion bounds for generalized Gaussian information sources and their application to image signals," Electronics Letters, vol. 16, no. 3, pp. 109-111, 1980.

[5] F. Abramovitch, T. Sapatinas, and B. Silverman, "Wavelet thresholding via a bayesian approach," Journal of the Royal Statistical Society, vol. 60, pp. 725-749, 1998.

[6] D. Leporini, J.-C. Pesquet, and H. Krim, "Best basis representations based on prior statistical models," in Bayesian Inference in Wavelet Based Models, ser. Lecture notes in statistics, P. Müller and B. Vidakovic, Eds. New-York: Springer-Verlag, 1999, no. 141, pp. 155-172.

[7] A. Gersho and R. Gray, Vector Quantization and Signal Compression. Boston, MA: Kluwer, 1992.

[8] J. Shapiro, "Embedded image coding using zerotrees of wavelet coefficients," IEEE Trans. on Signal Processing, vol. 41, no. 12, pp. 3445-3462, 1993.

[9] A. Said and W. A. Pearlman, "A new, fast, and efficient image codec based on set partitioning in hierarchical trees," IEEE Trans. Circuits and Systems for Video Technology, vol. 6, no. 3, pp. 243-249, 1996.

[10] S.-T. Hsiang and J. Wood, "Embedded image coding using zeroblocks of subband/wavelet coefficients and context modeling," in Proc. IEEE Int. Symp. Circuits Syst, ISCAS 2000 Geneva, vol. 3, Geneva, Switzerland, May 28-31 2000, pp. $662-665$.

[11] D. Taubman, "High performance scalable image compression with EBCOT," IEEE Trans. on Image Processing, vol. 9, no. 7, pp. 1158$1170,2000$.

[12] H. Gish and J. Pierce, "Asymptotically efficient quantizing," IEEE Trans. on Information Theory, vol. 14, no. 5, pp. 676-683, 1968. 
[13] P. Noll and R. Zelinski, "Bounds on quantizer performance in the low bit-rate region," IEEE Trans. on Communications, vol. 26, no. 2, pp. 300-304, 1978.

[14] N. Farvardin and J. W. Modestino, "Optimum quantizer performance for a class of non-Gaussian memoryless sources," IEEE Trans. on Information Theory, vol. 30, no. 3, pp. 485-497, 1984.

[15] A. György and T. Linder, "Optimal entropy-constrained scalar quantization of a uniform source," IEEE Trans. on Information Theory, vol. 46, no. 7, pp. 2704-2711, 2000.

[16] G. Sullivan, "Efficient scalar quantization of exponential and Laplacian random variables," IEEE Trans. on Information Theory, vol. 42, no. 5, pp. $1365-1374,1996$.

[17] D. Marco and D. Neuhoff, "Low-resolution scalar quantization for Gaussian sources and squared error," IEEE Trans. on Information Theory, vol. 52, no. 4, pp. 1689-1697, 2006.

[18] S. Jana and P. Moulin, "Optimality of KLT for high-rate transform coding of Gaussian vector-scale mixtures: Application to reconstruction, estimation, and classification," IEEE Trans. on Information Theory, vol. 52, no. 9, pp. 4049-4067, 2006.

[19] M. Pereira, M. Antonini, and M. Barlaud, "Multiple description image and video coding for wireless channels," Signal Process. Image Comm., vol. 18, pp. 925-945, 2003.

[20] J. Sun, W. Gao, D. Zhao, and Q. Huang, "Statistical model, analysis and approximation of rate-distortion function in MPEG-4 FGS videos," IEEE Trans. on Circuits and Systems for Video Technology, vol. 16, no. 4, pp. 535-539, 2006.

[21] M. Wang and M. Van Der Schaar, "Operational rate-distortion modeling for wavelet video coders," IEEE Trans. on Image Processing, vol. 54, no. 9, pp. 3505-3517, 2006.

[22] M. Gaubatz and S. Hemami, "Efficient entropy estimation based on doubly stochastic models for quantized wavelet image data," IEEE Trans. on Image Processing, vol. 16, no. 4, pp. 967-981, 2007.

[23] H. Rosenthal and J. Binia, “On the epsilon entropy of mixed random variables," IEEE Trans. on Information Theory, vol. 34, no. 5, pp. 1110-1114, 1988.

[24] A. György, T. Linder, and K. Zeger, "On the rate-distortion function of random vectors and stationary sources with mixed distributions," IEEE Trans. on Information Theory, vol. 45, no. 6, pp. 2110-2115, 1999.

[25] W. Gautschi, "The incomplete gamma functions since Tricomi," in Tricomi's ideas and contemporary applied mathematics, Atti dei Convegni Lincei, no. 147, Accademia Nazionale dei Lincei, Roma, 1998, pp. 203-237.

[26] D. Marco and D. Neuhoff, "The validity of the additive noise model for uniform scalar quantizers," IEEE Trans. on Information Theory, vol. 51, no. 5, pp. 1739-1755, 2005 .

[27] T. Cover and J. A. Thomas, Elements of Information Theory. New York: Wiley, 1991.

[28] Y. Meyer, Ondelettes et Opérateurs, Volume 1. Paris: Hermann, 1990.

[29] A. Chambolle, R. DeVore, N. Lee, and B. Lucier, "Nonlinear wavelet image processing: variational problems, compression, and noise removal through wavelet shrinkage," IEEE Trans. Image Process., vol. 7, no. 3, pp. 319-335, 1998.

[30] L. S. Gradshteyn and L. M. Ryzhik, Tables of Integrals, Series and Products. San Diego: Academic Press, 2000.

[31] T. Berger, Rate Distortion Theory. Englewood Cliffs, NJ: Prentice-Hall, 1971. 


\section{LIST OF FIGURES}

1 Entropy of a uniformly quantized GG source versus the normalized quantization step $\bar{q}$ when $\beta=1$ (top left), $\beta=4 / 3$ (top right), $\beta=5 / 3$ (bottom left) and $\beta=2$ (bottom right). $H_{f}$ is plotted in solid line, its lower approximation $H_{f}^{(2)}$ plotted in dotted line is almost perfectly superimposed on $H_{f}$ and the upper approximation $H_{f}^{(3)}+\Delta_{3}$ is plotted in dashdot line. . . . . . . . . . . . High and low resolution approximations of the entropy of a uniformly quantized GG source versus normalized quantization step $\bar{q}$ when $\beta=1$ (top), $\beta=3 / 2$ (middle) and $\beta=2$ (bottom). $H_{f}$ is plotted in solid line. Its high resolution approximation $\bar{q} \mapsto h_{\beta}(1)-\ln \bar{q}$ is plotted on the left in dashed line (for improved readibility, $\bar{q}$ is displayed in log scales on these graphs). Its low resolution approximations $\bar{q} \mapsto$ $-\mathrm{p}_{0} \ln \mathrm{p}_{0}-\left(1-\mathrm{p}_{0}\right) \ln \left(\left(1-\mathrm{p}_{0}\right) / 2\right)$ and $\bar{q} \mapsto \frac{\bar{q} \exp \left(-2^{-\beta} \bar{q}^{\beta}\right)}{2 \Gamma(1 / \beta) \tilde{q}^{\beta}}\left(1+(1-1 / \beta) \tilde{q}^{-2 \beta}\right)\left(2^{-\beta} \bar{q}^{\beta}+\ln \left(\tilde{q}^{\beta} / \bar{q}\right)+\ln (4 e \Gamma(1 / \beta))\right)$ are plotted on the right in dashdot line and dotted line, respectively.

3 High resolution (left) and low resolution (right) approximations of the entropy of a uniformly quantized BGG source versus the normalized quantization step $\bar{q}$ when $\epsilon=0.3, \beta=1$ (top), $\beta=$ $3 / 2$ (middle) and $\beta=2$ (bottom). $H_{f}$ is plotted in solid line, its high resolution approximation $\bar{q} \mapsto H_{\epsilon}+\epsilon\left(h_{\beta}(1)-\ln \bar{q}+\beta \bar{q} \ln \bar{q} /(2 \Gamma(1 / \beta))\right)$ is plotted in dashed line, its low resolution approximations $\bar{q} \mapsto-\left(1-\epsilon\left(1-\mathrm{p}_{0}\right)\right) \ln \left(1-\epsilon\left(1-\mathrm{p}_{0}\right)\right)-\epsilon\left(1-\mathrm{p}_{0}\right) \ln \left(\epsilon\left(1-\mathrm{p}_{0}\right) / 2\right)$ and $\bar{q} \mapsto$ $\frac{\epsilon \bar{q} \exp \left(-2^{-\beta} \bar{q}^{\beta}\right)}{2 \Gamma(1 / \beta) \tilde{q}^{\beta}}\left(1+(1-1 / \beta) \tilde{q}^{-2 \beta}\right)\left(2^{-\beta} \bar{q}^{\beta}+\ln \left(\tilde{q}^{\beta} / \bar{q}\right)+\ln (4 e \Gamma(1 / \beta) / \epsilon)\right)$ are plotted in dashdot line and dotted line, respectively. . . . . . . . . . . . . . . . . . . 34

4 Difference (in Nats) between the operational rate-distortion $R_{p}$ and the Shannon rate-distortion function $\mathcal{R}_{p}$ versus $p$ at low resolution for a GG source. . . . . . . . . . . . . . . . . 35

5 Distortion versus normalized quantization step $\bar{q}$ when $\beta=3 / 2$ and, $p=1$ (top), $p=3 / 2$ (middle) or $p=2$ (bottom). $\bar{d}_{p, 0}$ is plotted in solid line, its high and low resolution approximations are plotted in dashed and dashdot line, respectively. . . . . . . . . . . . . . . . . . . 36 

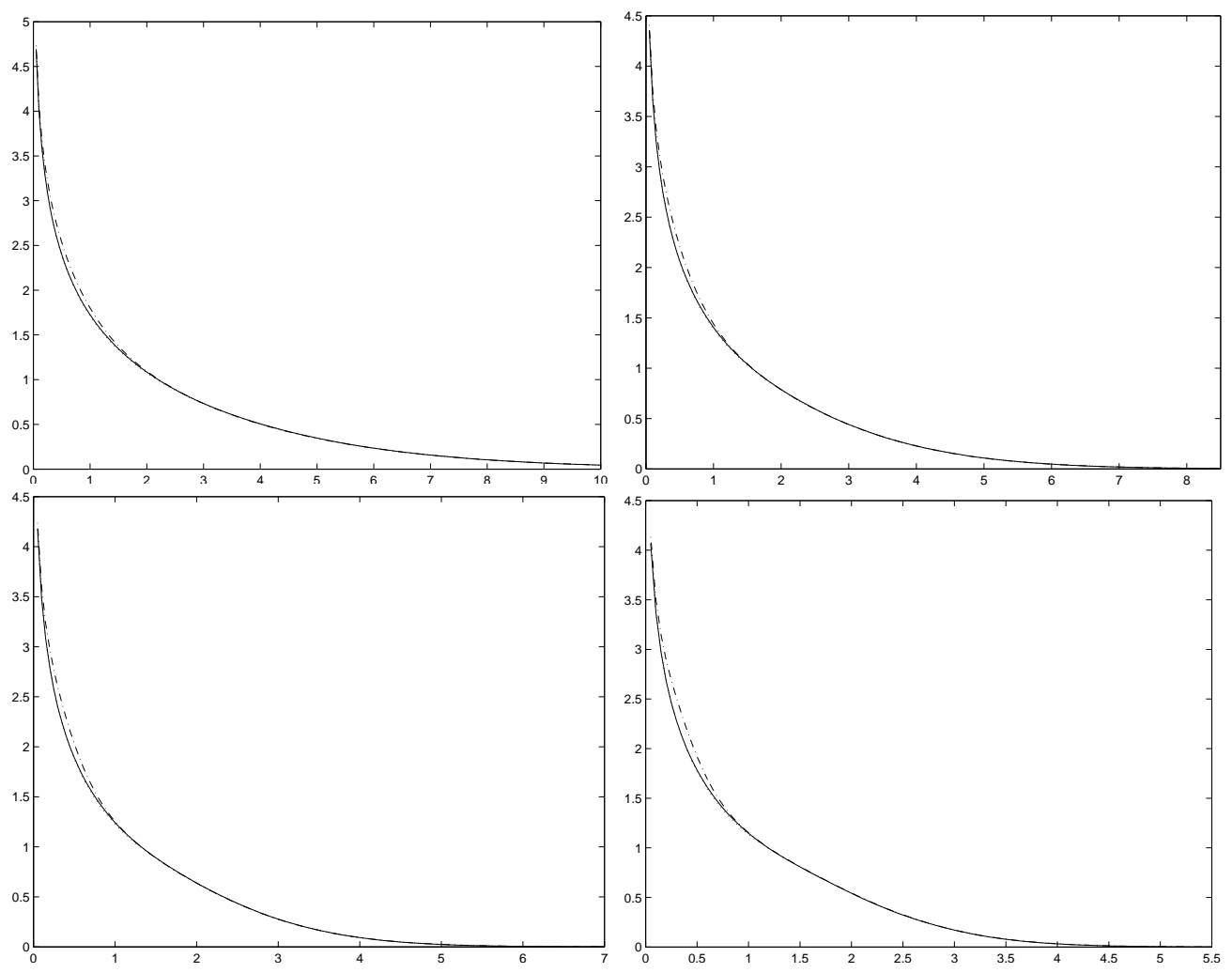

Fig. 1. Entropy of a uniformly quantized GG source versus the normalized quantization step $\bar{q}$ when $\beta=1$ (top left), $\beta=4 / 3$ (top right), $\beta=5 / 3$ (bottom left) and $\beta=2$ (bottom right). $H_{f}$ is plotted in solid line, its lower approximation $H_{f}^{(2)}$ plotted in dotted line is almost perfectly superimposed on $H_{f}$ and the upper approximation $H_{f}^{(3)}+\Delta_{3}$ is plotted in dashdot line. 

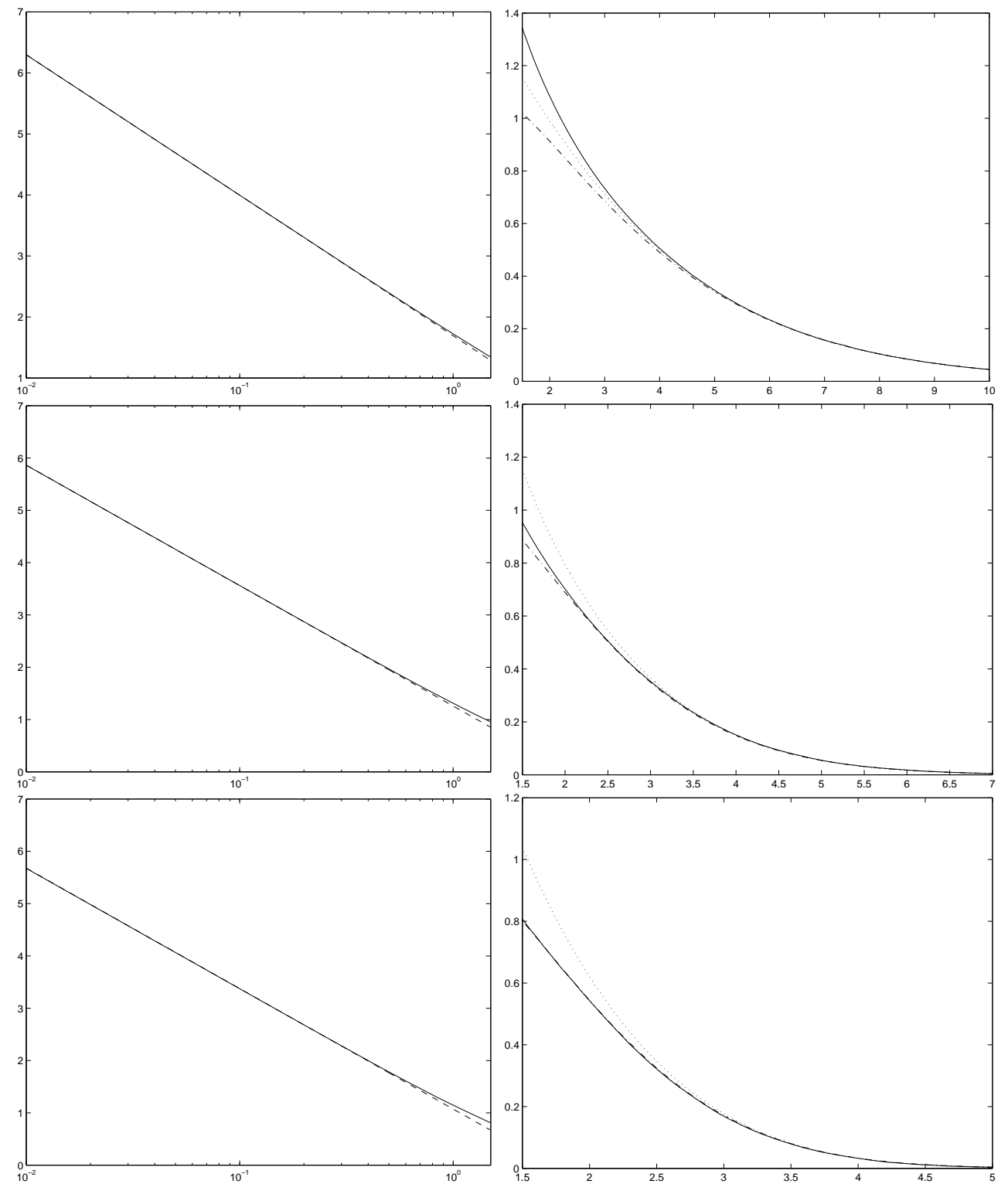

Fig. 2. High and low resolution approximations of the entropy of a uniformly quantized GG source versus normalized quantization step $\bar{q}$ when $\beta=1$ (top), $\beta=3 / 2$ (middle) and $\beta=2$ (bottom). $H_{f}$ is plotted in solid line. Its high resolution approximation $\bar{q} \mapsto h_{\beta}(1)-\ln \bar{q}$ is plotted on the left in dashed line (for improved readibility, $\bar{q}$ is displayed in log scales on these graphs). Its low resolution approximations $\bar{q} \mapsto-\mathrm{p}_{0} \ln \mathrm{p}_{0}-\left(1-\mathrm{p}_{0}\right) \ln \left(\left(1-\mathrm{p}_{0}\right) / 2\right)$ and $\bar{q} \mapsto \frac{\bar{q} \exp \left(-2^{-\beta} \bar{q}^{\beta}\right)}{2 \Gamma(1 / \beta) \tilde{q}^{\beta}}\left(1+(1-1 / \beta) \tilde{q}^{-2 \beta}\right)\left(2^{-\beta} \bar{q}^{\beta}+\ln \left(\tilde{q}^{\beta} / \bar{q}\right)+\ln (4 e \Gamma(1 / \beta))\right)$ are plotted on the right in dashdot line and dotted line, respectively. 

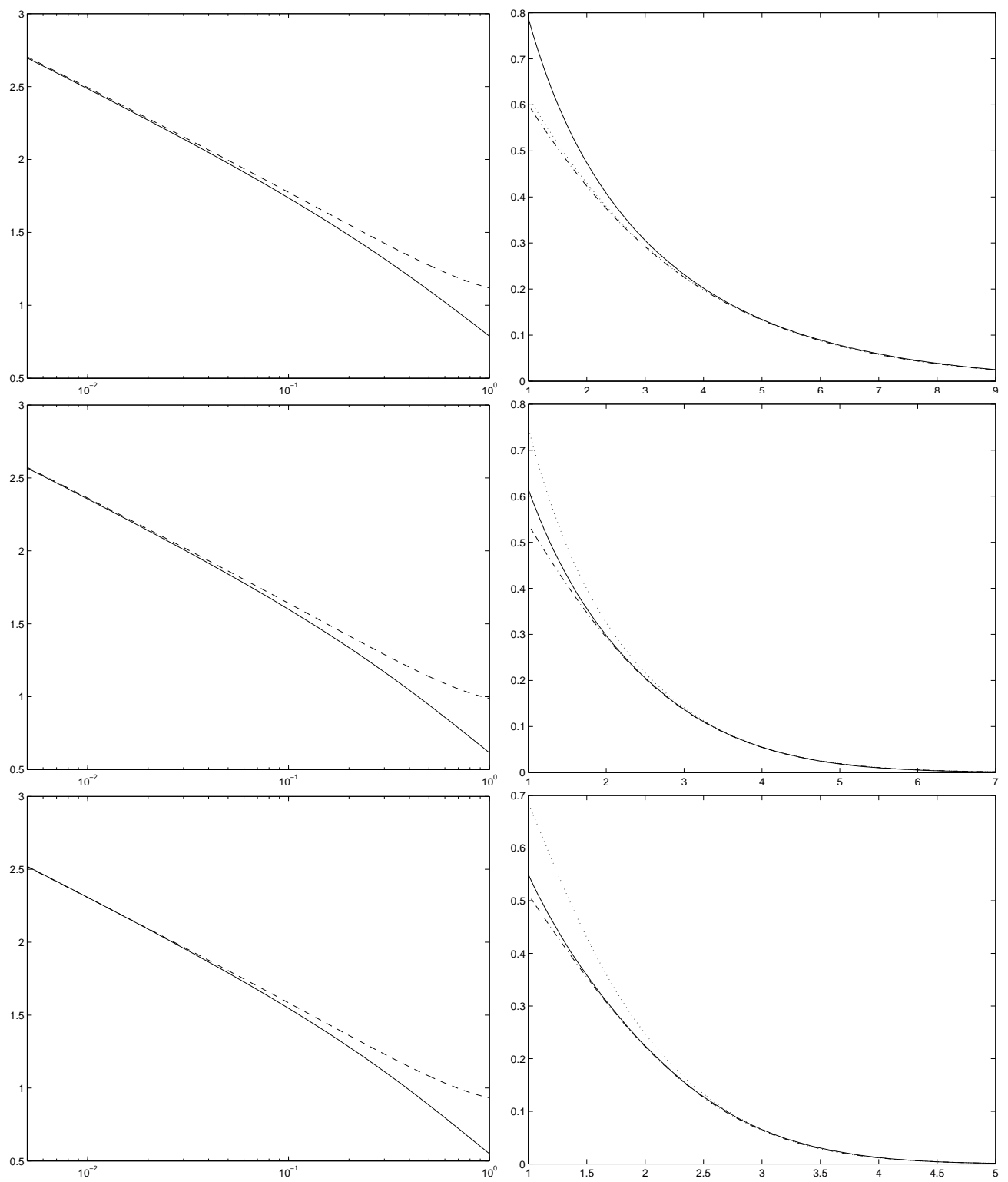

Fig. 3. High resolution (left) and low resolution (right) approximations of the entropy of a uniformly quantized BGG source versus the normalized quantization step $\bar{q}$ when $\epsilon=0.3, \beta=1$ (top), $\beta=3 / 2$ (middle) and $\beta=2$ (bottom). $H_{f}$ is plotted in solid line, its high resolution approximation $\bar{q} \mapsto H_{\epsilon}+\epsilon\left(h_{\beta}(1)-\ln \bar{q}+\beta \bar{q} \ln \bar{q} /(2 \Gamma(1 / \beta))\right)$ is plotted in dashed line, its low resolution approximations $\bar{q} \mapsto-\left(1-\epsilon\left(1-\mathrm{p}_{0}\right)\right) \ln (1-$ $\left.\epsilon\left(1-\mathrm{p}_{0}\right)\right)-\epsilon\left(1-\mathrm{p}_{0}\right) \ln \left(\epsilon\left(1-\mathrm{p}_{0}\right) / 2\right)$ and $\bar{q} \mapsto \frac{\epsilon \bar{q} \exp \left(-2^{-\beta} \bar{q}^{\beta}\right)}{2 \Gamma(1 / \beta) \tilde{q}^{\beta}}\left(1+(1-1 / \beta) \tilde{q}^{-2 \beta}\right)\left(2^{-\beta} \bar{q}^{\beta}+\ln \left(\tilde{q}^{\beta} / \bar{q}\right)+\ln (4 e \Gamma(1 / \beta) / \epsilon)\right)$ are plotted in dashdot line and dotted line, respectively. 


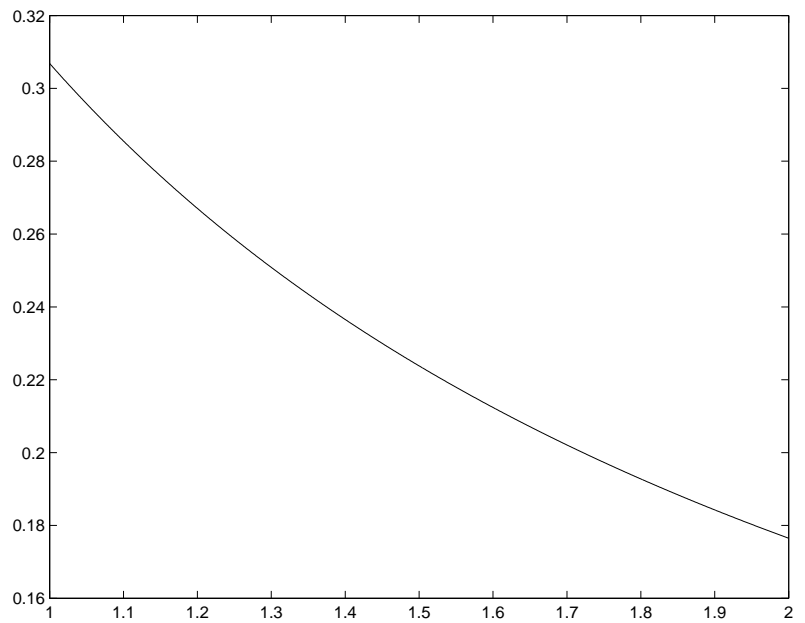

Fig. 4. Difference (in Nats) between the operational rate-distortion $R_{p}$ and the Shannon rate-distortion function $\mathcal{R}_{p}$ versus $p$ at low resolution for a GG source. 


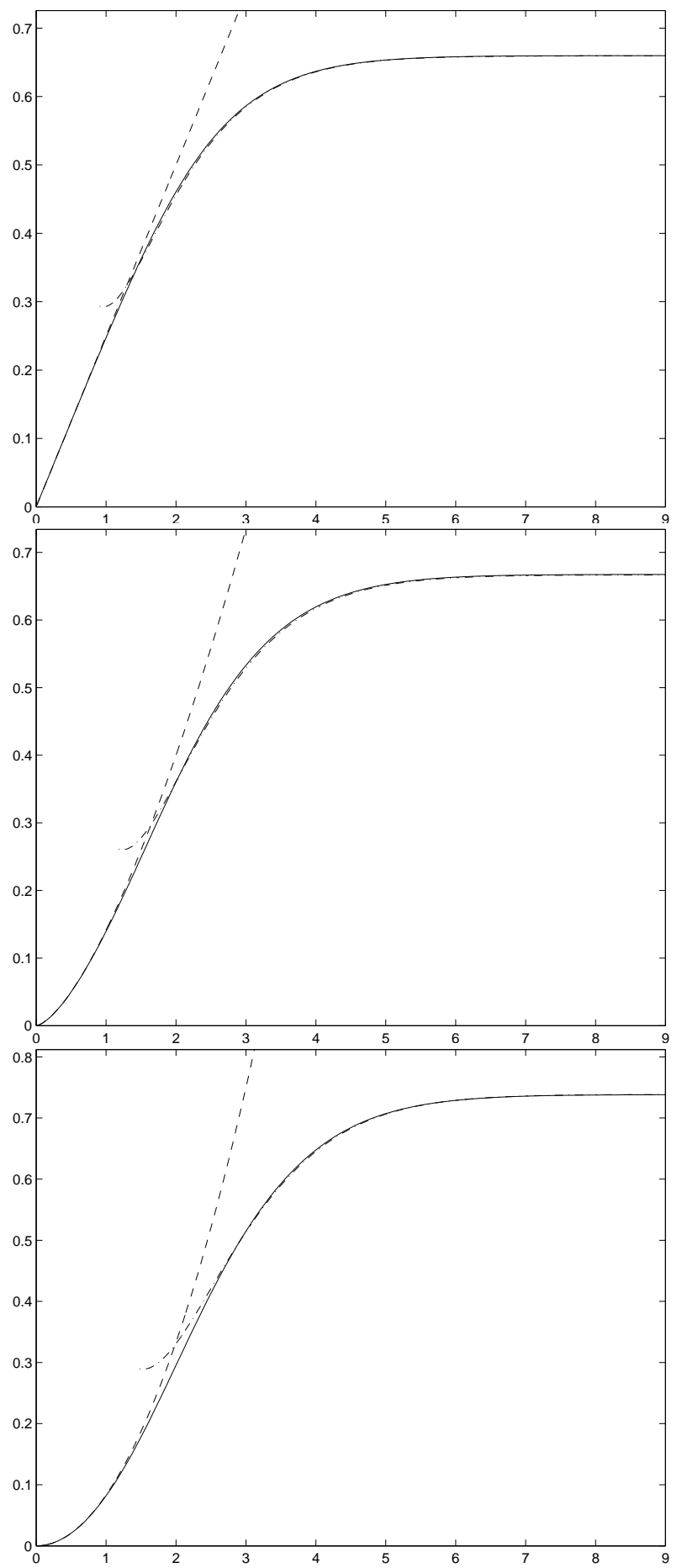

Fig. 5. Distortion versus normalized quantization step $\bar{q}$ when $\beta=3 / 2$ and, $p=1$ (top), $p=3 / 2$ (middle) or $p=2$ (bottom). $\bar{d}_{p, 0}$ is plotted in solid line, its high and low resolution approximations are plotted in dashed and dashdot line, respectively. 